\title{
SINGULARITIES OF EDDY CURRENT PROBLEMS
}

\author{
Martin Costabel $^{1}$, Monique Dauge ${ }^{1}$ and Serge Nicaise ${ }^{2}$
}

\begin{abstract}
We consider the time-harmonic eddy current problem in its electric formulation where the conductor is a polyhedral domain. By proving the convergence in energy, we justify in what sense this problem is the limit of a family of Maxwell transmission problems: Rather than a low frequency limit, this limit has to be understood in the sense of Bossavit [11]. We describe the singularities of the solutions. They are related to edge and corner singularities of certain problems for the scalar Laplace operator, namely the interior Neumann problem, the exterior Dirichlet problem, and possibly, an interface problem. These singularities are the limit of the singularities of the related family of Maxwell problems.
\end{abstract}

Mathematics Subject Classification. 35B65, 35R05, 35Q60.

Received: May 20, 2003.

\section{Maxwell Equations And the edDy CURRent Limit}

Let us consider the model case of an homogeneous conducting body $\Omega_{C}$ which we assume to be a threedimensional bounded polyhedral domain with a Lipschitz boundary $B$. The conductivity $\sigma=\sigma_{C}$ is constant and positive inside $\Omega_{C}$, while $\sigma$ vanishes outside $\Omega_{C}$, i.e., $\sigma \equiv 0$ in the "air" (or "empty") region $\Omega_{E}=\mathbb{R}^{3} \backslash \bar{\Omega}_{C}$. For the sake of simplicity we further assume that the boundary $B$ of $\Omega_{C}$ is connected ${ }^{(*)}$. The electric permittivity $\varepsilon$ is equal to a positive constant $\varepsilon_{C}$ inside $\Omega_{C}$ and has another value $\varepsilon_{E}$ in the exterior medium. Similarly, the magnetic permeability $\mu$ is equal to $\mu_{C}>0$ in $\Omega_{C}$ and to $\mu_{E}>0$ in $\Omega_{E}$. The treatment of piecewise constant $\sigma_{C}, \varepsilon_{C}, \mu_{C}$ and $\mu_{E}$ can be made in a similar manner.

\subsection{Maxwell and eddy current problems}

Let $\omega>0$ be a fixed frequency. The time harmonic Maxwell equations are

$$
\begin{aligned}
\operatorname{curl} \mathbf{E} & =-i \omega \mu \mathbf{H} \quad \text { in } \mathbb{R}^{3}, \\
\operatorname{curl} \mathbf{H} & =(i \omega \varepsilon+\sigma) \mathbf{E}+\mathbf{j}_{0} \quad \text { in } \mathbb{R}^{3},
\end{aligned}
$$

Keywords and phrases. Eddy current problem, corner singularity, edge singularity.

1 IRMAR, Université de Rennes 1, Campus de Beaulieu, 35042 Rennes Cedex, France.

http://perso.univ-rennes1.fr/martin.costabel, http://perso.univ-rennes1.fr/monique.dauge

2 Université de Valenciennes et du Hainaut Cambrésis, MACS, Le Mont Houy, 59313 Valenciennes Cedex 9, France.

http://www .univ-valenciennes.fr/macs/Serge.Nicaise

${ }^{(*)}$ The issue of a multiple connected $B$ is independent of the question of singularities. We will just mention the modifications necessary when $B$ is multiply connected, see Remark 2.1 at the end of Section 2 . 
$\mathbf{E}$ (resp. $\mathbf{H}$ ) is the electric (resp. magnetic) field and $\mathbf{j}_{0}$ is the source current density which is supposed to be a $L^{2}\left(\mathbb{R}^{3}\right)$ field with support in $\Omega_{C}$ and to be divergence free, i.e. $\operatorname{div} \mathbf{j}_{0}=0$ in $\mathbb{R}^{3}$. Let us recall

Lemma 1.1. Let $\mathbf{u} \in L^{2}\left(\mathbb{R}^{3}\right)^{3}$ be such that $\left.\mathbf{u}\right|_{\Omega_{E}} \equiv 0$ and $\operatorname{div} \mathbf{u}=0$ in $\mathbb{R}^{3}$. Then the normal trace $\left.\mathbf{u}\right|_{\Omega_{C}} \cdot \mathbf{n}$ on $B$ is zero (here $\mathbf{n}$ denotes the unit outward normal vector on $B$, pointing from $\Omega_{C}$ to $\Omega_{E}$ ).

Thus the assumption on $\operatorname{div} \mathbf{j}_{0}$ is equivalent to

$$
\operatorname{div} \mathbf{j}_{0}=0 \text { in } \Omega_{C} \quad \text { and } \quad \mathbf{j}_{0} \cdot \mathbf{n}=0 \text { on } B .
$$

Note that, taking the divergence of equation (2), we obtain the following equation on the divergence of $\mathbf{E}$ :

$$
\operatorname{div}(i \omega \varepsilon+\sigma) \mathbf{E}=0 \quad \text { in } \mathbb{R}^{3} .
$$

Equations (1) - (2) have to be completed by conditions at infinity (Silver-Müller radiation conditions)

$$
\lim _{|x| \rightarrow \infty}(\mathbf{H} \times x-|x| \mathbf{E})=0 .
$$

The time-harmonic eddy current problem $[3,10,11,22]$ reads

$$
\begin{aligned}
& \operatorname{curl} \mathbf{E}=-i \omega \mu \mathbf{H} \quad \text { in } \mathbb{R}^{3}, \\
& \operatorname{curl} \mathbf{H}=\sigma \mathbf{E}+\mathbf{j}_{0} \quad \text { in } \mathbb{R}^{3} .
\end{aligned}
$$

Let us denote $\left.\mathbf{E}\right|_{\Omega_{C}}$ and $\left.\mathbf{E}\right|_{\Omega_{E}}$ by $\mathbf{E}_{C}$ and $\mathbf{E}_{E}$, respectively. Now, taking the divergence of equation (6) we only obtain, thanks to Lemma 1.1, $\operatorname{div} \mathbf{E}_{C}=0$ in $\Omega_{C}$ and $\mathbf{E}_{C} \cdot \mathbf{n}=0$ on $B$. These conditions have to be completed by the gauge conditions:

$$
\operatorname{div} \mathbf{E}_{E}=0 \text { in } \Omega_{E} \text { and } \int_{B} \mathbf{E}_{E} \cdot \mathbf{n} \mathrm{d} S=0 .
$$

The condition at infinity takes the form

$$
\mathbf{E}(x)=O\left(|x|^{-1}\right), \quad \mathbf{H}(x)=O\left(|x|^{-1}\right) \quad \text { as } \quad|x| \rightarrow \infty .
$$

Remark 1.1. Equations (5)-(6) are clearly obtained from (1)-(2) by setting $\varepsilon$ to zero. The gauge conditions (7) can also be obtained from (3): Since $i \omega \varepsilon+\sigma$ is equal to the two non-zero constants $i \omega \varepsilon_{C}+\sigma$ in $\Omega_{C}$ and $i \omega \varepsilon_{E}$ in $\Omega_{E}$, (3) implies that $\operatorname{div} \mathbf{E}_{C}=0$ in $\Omega_{C}$, div $\mathbf{E}_{E}=0$ in $\Omega_{E}$ and (by a result similar to Lem. 1.1)

$$
\left(i \omega \varepsilon_{C}+\sigma_{C}\right) \mathbf{E}_{C} \cdot \mathbf{n}=i \omega \varepsilon_{E} \mathbf{E}_{E} \cdot \mathbf{n} \quad \text { on } \quad B .
$$

The condition $\operatorname{div} \mathbf{E}_{C}=0$ implies by integration by parts that $\int_{B} \mathbf{E}_{C} \cdot \mathbf{n}=0$. Then, by (9), we obtain that

$$
\int_{B} \mathbf{E}_{E} \cdot \mathbf{n} \mathrm{d} S=0 .
$$

Setting $\varepsilon_{C}=\varepsilon_{E}=0$, we obtain (7) and the two conditions issued from the equation $\operatorname{div}(\sigma \mathbf{E})=0$, that is

$$
\operatorname{div} \mathbf{E}=0 \text { in } \Omega_{C} \cup \Omega_{E} \text { and } \mathbf{E}_{C} \cdot \mathbf{n}=0 \text { on } B .
$$

Thus we see that the gauge conditions (7) are natural. But we obtain them by first deducing conditions on the divergence of the Maxwell solution $\mathbf{E}$ and then passing to the limit. The converse order does not provide (7). 
Remark 1.2. The conditions at infinity (4) imply the uniqueness of solutions for equations (1)-(2) (Rellich lemma). Moreover, with the (exterior) wave number $k:=\omega \sqrt{\varepsilon_{E} \mu_{E}}$, we have the following asymptotics at infinity (here $\hat{x}:=x /|x|)$ :

$$
\mathbf{E}(x)=\frac{\mathrm{e}^{\mathrm{i} k|x|}}{|x|}\left(\mathbf{E}^{\infty}(\hat{x})+O\left(|x|^{-1}\right)\right) \quad \text { as } \quad|x| \rightarrow \infty .
$$

The function $\mathbf{E}^{\infty}$ is the electric far field pattern, see [12].

Concerning equations (5)-(7), the conditions at infinity (8) also imply the uniqueness, and the following asymptotics at infinity holds [3, Prop. 3.1]

$$
\mathbf{E}(x)=O\left(|x|^{-2}\right), \quad \mathbf{H}(x)=O\left(|x|^{-2}\right) \quad \text { as } \quad|x| \rightarrow \infty .
$$

This means that the far field pattern goes to zero in the eddy current limit.

\subsection{Eddy current limit}

We want to give a sense to the notion of eddy current limit: This means that the quantities $\omega \varepsilon_{C} / \sigma_{C}$ and $\omega \varepsilon_{E} / \sigma_{C}$ are small. For a conducting material, the permittivity $\varepsilon_{C}$ is of the same order of magnitude than $\varepsilon_{E}$ (also denoted $\varepsilon_{0}$ ), but $\varepsilon_{C} / \sigma_{C}$ is very small. For moderate frequencies $\omega$ the quantities $\omega \varepsilon_{C} / \sigma_{C}$ and $\omega \varepsilon_{E} / \sigma_{C}$ are still small. Let us fix two numbers $\hat{\varepsilon}_{C}$ and $\hat{\varepsilon}_{E}$ which are of the same order than $\sigma_{C}$ and such that there exists $\delta>0$ (thus $\delta$ is small)

Thus

$$
\varepsilon_{C}=\delta \hat{\varepsilon}_{C} \quad \text { and } \quad \varepsilon_{E}=\delta \hat{\varepsilon}_{E}
$$

$$
i \omega \varepsilon+\sigma= \begin{cases}i \omega \delta \hat{\varepsilon}_{C}+\sigma_{C} & \text { in } \Omega_{C} \\ i \omega \delta \hat{\varepsilon}_{E} & \text { in } \Omega_{E} .\end{cases}
$$

We fix $\sigma_{C}, \omega, \hat{\varepsilon}_{C}$ and $\hat{\varepsilon}_{E}$. The eddy current limit is the limit as $\delta \rightarrow 0$. This notion of limit coincides with that presented in [11, Ch. 4].

Thus, we may say that this limit is a "low frequency limit" only in the special sense that it is not a high frequency limit. This limit is not a limit as $\omega \rightarrow 0$. This fact is important, since there is a notion of high frequency asymptotics inside the eddy current model, which gives rise to boundary layers inside the conductor (skin effect).

\subsection{Outline of the paper}

In this paper, our main goal is the description of the singularities near the edges and corners of $B$ of the eddy current problem (5)-(8). Moreover, considering a one parameter family of Maxwell problems along the lines of (12)-(13), we want to follow the singularities as $\delta \rightarrow 0$. The "standard" regularity and singularity results for the Maxwell interface problem from $[9,15,17]$ can be adapted for $\delta>0$, but not for the limit $\delta=0$.

We show here that the regularity and the singularities of the solution of the eddy current problem are related to the regularity and the singularities of the interior Neumann Laplace operator, the exterior Dirichlet Laplace operator and the interface Laplace operator (for the parameter $\mu$ ). To our knowledge this coupling phenomenon seems to be new. As in $[15,17]$ our technique relies on a regularized formulation of the problem and on the use of Mellin transformation.

Such results are useful for the numerical analysis of the eddy current problem as considered in [1,22], where certain refinement rules or weighted regularization are susceptible to give a better order of convergence $[6,16,19,29]$.

Moreover, we show how the singularities of the eddy current problem are the limit as $\delta \rightarrow 0$ of the singularities of the Maxwell problem.

It turns out that from the point of view of singularities, the eddy current limit $\delta \rightarrow 0$ behaves like a regular perturbation problem. This means that one can choose the singular functions in such a way that they depend analytically on $\delta$ for $\delta$ in a neighborhood of 0 , see Section 7. It does not mean, however, that the regularity of the solution as measured by Sobolev regularity in $\Omega_{C}$ (or in $\Omega_{E}$ ) is a continuous function of $\delta$ : Indeed, if 
the conductor is convex, the electric field $\mathbf{E}_{C}$ in the eddy current model will be a bounded function inside the conductor, whereas the exterior electric field $\mathbf{E}_{E}$ will be unbounded, in general. In the full Maxwell interface problem, i.e. for any $\delta>0$, both parts $\mathbf{E}_{C}$ and $\mathbf{E}_{E}$ of the field will be unbounded, in general. In terms of Sobolev indices, the regularity of $\mathbf{E}_{C}$ may jump from $H^{s}$ with $\frac{1}{2}<s<1$ to more than $H^{1}$ regularity as $\delta \rightarrow 0$.

Here is the outline of our paper: Since we are mainly interested in the singularities near $B$, and since their structure is of local nature, we will define our one-parameter family of problems in a bounded domain $\Omega$ and work in that framework in the remainder of the paper. In Section 2 we first replace the problems in $\mathbb{R}^{3}$ with problems in $\Omega$, we propose equivalent regularized variational formulations and we prove the convergence of solutions in the energy space in the eddy current limit, i.e. as $\delta \rightarrow 0$.

Section 3 is devoted to a splitting of the variational space into a regular vector field which is piecewise $H^{1}$ and a singular part which is the gradient of a singular solution of a Laplace interface problem; this kind of decomposition is in the spirit of [5,7-9].

After a short description of the corner and edge singularities for the Laplace interface problem in Section 4, we start the analysis of their dependence on the parameter $\delta$ and prove that their exponents (degrees) depend continuously on $\delta$ up to the limit $\delta=0$.

We describe in Section 5 the corner and edge singularities for our eddy current problem (case when $\delta=0$, the case $\delta>0$ being already investigated in [17]). Section 6 is devoted to the regularity of the solution of the eddy current problem in terms of standard Sobolev spaces, we further give two different decompositions into a regular part and a singular one.

Finally Section 7 analyzes the continuous dependence of the singular functions on the parameter $\delta$ using Mellin symbols and the Cauchy residue formula.

For $D$ a subdomain of $\mathbb{R}^{3}$ we denote by $H^{s}(D)$ the standard Sobolev space of order $s$, with norm denoted by $\|\cdot\|_{s, D}$.

\section{VARIATIONAL FORMULATIONS}

Let us take the polyhedron $\Omega_{C}$ with connected boundary $B$ as in the previous section and let $\Omega$ be a smooth domain with trivial topology (for example a ball) which contains $\bar{\Omega}_{C}$. Now the exterior domain $\Omega_{E}$ is defined as $\Omega_{E}=\Omega \backslash \bar{\Omega}_{C}$.

For a function $u$ defined in $\Omega$ we set $u_{C}$ (resp. $u_{E}$ ) its restriction to $\Omega_{C}$ (resp. $\Omega_{E}$ ). For a function $u$ defined near $B$ and such that the traces of $u_{C}$ and of $u_{E}$ on $B$ have a meaning, we set $[u]=u_{C}-u_{E}$ its jump through $B$.

The partial differential operator $\partial_{n}$ defined on $B$ is the unit normal derivative pointing from $\Omega_{C}$ to $\Omega_{E}$.

\subsection{Strong form of equations}

Instead of conditions at infinity (4) or (8), we will simply impose the perfect conductor boundary conditions on the exterior boundary $\partial \Omega$.

According to (13), we set $\hat{\varepsilon}=\hat{\varepsilon}_{C}$ in $\Omega_{C}$ and $\hat{\varepsilon}=\hat{\varepsilon}_{E}$ in $\Omega_{E}$. Our Maxwell problem with parameter $\delta$ is

$$
\left\{\begin{aligned}
\operatorname{curl} \mathbf{E}^{\delta} & =-i \omega \mu \mathbf{H}^{\delta} & & \text { in } \quad \Omega, \\
\operatorname{curl} \mathbf{H}^{\delta} & =(i \omega \delta \hat{\varepsilon}+\sigma) \mathbf{E}^{\delta}+\mathbf{j}_{0} & & \text { in } \quad \Omega, \\
\mathbf{E}^{\delta} \times \mathbf{n} & =0 \quad \text { and } \quad \mathbf{H}^{\delta} \cdot \mathbf{n}=0 & & \text { on } \quad \partial \Omega,
\end{aligned}\right.
$$

whereas the eddy current problem is

$$
\left\{\begin{aligned}
\operatorname{curl} \mathbf{E}^{0} & =-i \omega \mu \mathbf{H}^{0} & & \text { in } \Omega, \\
\operatorname{curl} \mathbf{H}^{0} & =\sigma \mathbf{E}^{0}+\mathbf{j}_{0} & & \text { in } \Omega, \\
\operatorname{div} \mathbf{E}^{0} & =0 & & \text { in } \Omega_{E} \\
\int_{B} \mathbf{E}_{E}^{0} \cdot \mathbf{n} \mathrm{d} S & =0 & & \\
\mathbf{E}^{0} \times \mathbf{n} & =0 \quad \text { and } \quad \mathbf{H}^{0} \cdot \mathbf{n}=0 & & \text { on } \quad \partial \Omega
\end{aligned}\right.
$$


The resolution of the last problem is usually made by eliminating either the electric field (H-formulation or magnetic approach $[2,10,11])$ or the magnetic field (E-formulation or electric approach $[1,3,10,11,22])$. Here we focus on the electric approach, for both (14) and (15). We find the following systems of equations for any $\delta$. This includes for $\delta \geq 0$ both the Maxwell and the eddy current problems.

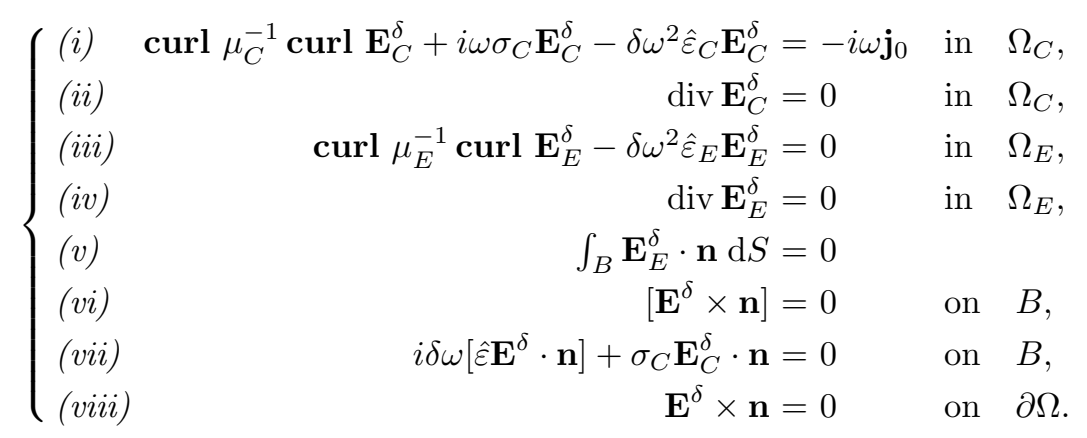

The magnetic field is then given by $\mathbf{H}^{\delta}=\frac{i}{\omega \mu} \mathbf{c u r l} \mathbf{E}^{\delta}$ in $\Omega$.

\subsection{Variational space and forms}

We now propose a variational space suitable for a regularized formulation, and independent of $\delta$, i.e. suitable both for the Maxwell and eddy current problems. Let $\mathbf{H}_{0}(\mathbf{c u r l}, \Omega)$ be the standard space

$$
\mathbf{H}_{0}(\mathbf{c u r l}, \Omega)=\left\{\mathbf{u} \in L^{2}(\Omega)^{3}: \operatorname{curl} \mathbf{u} \in L^{2}(\Omega)^{3}, \quad \mathbf{u} \times \mathbf{n}=0 \text { on } \partial \Omega\right\} .
$$

Our variational space is $\mathbf{Y}(\Omega)$ defined as

$$
\mathbf{Y}(\Omega)=\left\{\mathbf{u} \in \mathbf{H}_{0}(\operatorname{curl}, \Omega): \operatorname{div} \mathbf{u}_{C} \in L^{2}\left(\Omega_{C}\right), \operatorname{div} \mathbf{u}_{E} \in L^{2}\left(\Omega_{E}\right), \int_{B} \mathbf{u}_{E} \cdot \mathbf{n}=0\right\}
$$

equipped with the norm

$$
\|\mathbf{u}\|_{\mathbf{Y}(\Omega)}^{2}=\|\mathbf{u}\|_{0, \Omega}^{2}+\|\operatorname{curl} \mathbf{u}\|_{0, \Omega}^{2}+\left\|\operatorname{div} \mathbf{u}_{C}\right\|_{0, \Omega_{C}}^{2}+\left\|\operatorname{div} \mathbf{u}_{E}\right\|_{0, \Omega_{E}}^{2} .
$$

The gradient fields belonging to $\mathbf{Y}(\Omega)$ are associated with potentials $\varphi$ in the space

$$
\begin{aligned}
\left\{\varphi \in L^{2}(\Omega):\right. & \varphi_{C} \in H^{1}\left(\Omega_{C}\right), \varphi_{E} \in H^{1}\left(\Omega_{E}\right), \\
& {[\varphi]=c_{1} \text { on } B, \varphi=0 \text { on } \partial \Omega, \quad c_{1} \in \mathbb{C} } \\
& \left.\Delta \varphi_{C} \in L^{2}\left(\Omega_{C}\right), \quad \Delta \varphi_{E} \in L^{2}\left(\Omega_{E}\right), \quad \int_{B} \partial_{n} \varphi_{E} \mathrm{~d} S=0\right\} .
\end{aligned}
$$

For such potentials, the associated field in $\mathbf{Y}(\Omega)$ is the "broken" gradient field $\widetilde{\nabla} \varphi \in L^{2}(\Omega)^{3}$ defined as

$$
\left.(\widetilde{\nabla} \varphi)\right|_{\Omega_{C}}=\nabla \varphi_{C} \quad \text { and }\left.\quad(\widetilde{\nabla} \varphi)\right|_{\Omega_{E}}=\nabla \varphi_{E}
$$

The following result on potentials in the exterior part $\Omega_{E}$ will be used several times. Note that $B$ and $\partial \Omega$ are the two components of the boundary $\partial \Omega_{E}$ of $\Omega_{E}$. 
Lemma 2.1. For any $f \in L^{2}\left(\Omega_{E}\right), v \in H^{1 / 2}(B)$ and $b \in \mathbb{C}$, there exists a unique solution $\varphi \in H^{1}\left(\Omega_{E}\right)$ of the following boundary value problem

$$
\left\{\begin{aligned}
\Delta \varphi & =f \text { in } \Omega_{E}, \\
\varphi & =0 \text { on } \partial \Omega, \quad \varphi=v+c \text { on } B \quad \text { for some } c \in \mathbb{C} \\
\int_{B} \partial_{n} \varphi \mathrm{d} S & =b
\end{aligned}\right.
$$

There is an estimate

$$
\|\varphi\|_{1, \Omega_{E}} \leq C\left(\|f\|_{0, \Omega_{E}}+\|v\|_{H^{1 / 2}(B) / \mathbb{C}}+|b|\right) .
$$

Proof. Let $\varphi_{0}$ be the solution of the Dirichlet problem $\Delta \varphi_{0}=f$ in $\Omega_{E}, \varphi_{0}=0$ on $\partial \Omega$ and $\varphi_{0}=v$ on $B$.

Let $q$ be the solution of the problem $\Delta q=0$ in $\Omega_{E}, q=0$ on $\partial \Omega, q=$ constant on $B$ and $\int_{B} \partial_{n} q=1$ (compare with [4, Prop. 3.18]).

With $\ell=\int_{B} \partial_{n} \varphi_{0}$, the function $\varphi:=\varphi_{0}+(b-\ell) q$ is the solution of (18).

For the estimate (19), one notes that

$$
\left\|\varphi_{0}\right\|_{1, \Omega_{E}}+|\ell| \leq C_{1}\left(\|f\|_{0, \Omega_{E}}+\|v\|_{1 / 2, B}\right)
$$

and hence

$$
\begin{aligned}
\|\varphi\|_{1, \Omega_{E}} & \leq\left\|\varphi_{0}\right\|_{1, \Omega_{E}}+(|\ell|+|b|)\|q\|_{1, \Omega_{E}} \\
& \leq C_{2}\left(\|f\|_{0, \Omega_{E}}+\|v\|_{1 / 2, B}+|b|\right)
\end{aligned}
$$

with $C_{2}=\max \left\{C_{1}, C_{1}\|q\|_{1, \Omega_{E}},\|q\|_{1, \Omega_{E}}\right\}$.

We can replace $\|v\|_{H^{1 / 2}(B)}$ by $\|v\|_{H^{1 / 2}(B) / \mathbb{C}}$ here because $\varphi$ depends only on $v$ modulo the constants.

Let us further define the following bilinear form on $\mathbf{Y}(\Omega)$ : For $\mathbf{u}, \mathbf{v} \in \mathbf{Y}(\Omega)$ :

$$
a^{\delta}(\mathbf{u}, \mathbf{v})=\int_{\Omega}\left(\mu^{-1} \operatorname{curl} \mathbf{u} \cdot \operatorname{curl} \overline{\mathbf{v}}-\delta \omega^{2} \hat{\varepsilon} \mathbf{u} \cdot \overline{\mathbf{v}}\right) \mathrm{d} x+i \omega \int_{\Omega_{C}} \sigma_{C} \mathbf{u} \cdot \overline{\mathbf{v}} \mathrm{d} x
$$

and its regularized version

$$
a_{R}^{\delta}(\mathbf{u}, \mathbf{v})=a^{\delta}(\mathbf{u}, \mathbf{v})+\int_{\Omega_{C}} \operatorname{div} \mathbf{u}_{C} \operatorname{div} \overline{\mathbf{v}}_{C} \mathrm{~d} x+\int_{\Omega_{E}} \operatorname{div} \mathbf{u}_{E} \operatorname{div} \overline{\mathbf{v}}_{E} \mathrm{~d} x .
$$

Lemma 2.2. Let the positive constants $\mu_{C}, \mu_{E}, \hat{\varepsilon}_{C}, \hat{\varepsilon}_{E}, \sigma_{C}$ and $\omega$ be fixed. Then there exists $\delta_{0}>0$ such that for all $\delta \in\left[0, \delta_{0}\right], a_{R}^{\delta}$ is strongly coercive on $\mathbf{Y}(\Omega): \exists \alpha \in \mathbb{C}, \exists c_{0}>0, \forall \delta \in\left[0, \delta_{0}\right], \forall \mathbf{u} \in \mathbf{Y}(\Omega)$

$$
\operatorname{Re}\left(\alpha a_{R}^{\delta}(\mathbf{u}, \mathbf{u})\right) \geq c_{0}\|\mathbf{u}\|_{\mathbf{Y}(\Omega)}^{2}
$$

Proof. Since $\left|a_{R}^{\delta}(\mathbf{u}, \mathbf{u})-a_{R}^{0}(\mathbf{u}, \mathbf{u})\right| \leq C \delta\|\mathbf{u}\|_{0, \Omega}^{2} \leq C \delta\|\mathbf{u}\|_{\mathbf{Y}(\Omega)}^{2}$, it is clearly enough to prove the coerciveness property for $\delta=0$ : We check that if the coerciveness estimate (16) holds for $\delta=0$ with the constant $c_{0}$, then it holds for any $\delta \in\left[0, \delta_{0}\right]$ with $\delta_{0}=c_{0} / 2 C$ and with $c_{0} / 2$ instead of $c_{0}$.

Let us take $\alpha=\mathrm{e}^{-\mathrm{i} \pi / 4}$. Then

$$
\operatorname{Re}\left(\alpha a_{R}^{0}(\mathbf{u}, \mathbf{u})\right) \gtrsim\|\mathbf{c u r l} \mathbf{u}\|_{0, \Omega}^{2}+\|\mathbf{u}\|_{0, \Omega_{C}}^{2}+\|\operatorname{div} \mathbf{u}\|_{0, \Omega_{C}}^{2}+\|\operatorname{div} \mathbf{u}\|_{0, \Omega_{E}}^{2}
$$

It remains to prove that the right hand side above is an upper bound for $\|\mathbf{u}\|_{0, \Omega_{E}}^{2}$. 
Let $\mathbf{w} \in H^{1}(\Omega)^{3}$ be such that $\operatorname{curl} \mathbf{w}=\mathbf{c u r l} \mathbf{u}$ in $\Omega$ and $\mathbf{w} \times \mathbf{n}=0$ on $\partial \Omega$. This exists according to [4, Lem. 3.5] and can be chosen such that $\operatorname{div} \mathbf{w}=0$ in $\Omega$, with the estimate

$$
\|\mathbf{w}\|_{1, \Omega} \lesssim\|\operatorname{curl} \mathbf{u}\|_{0, \Omega}
$$

Since $\Omega$ is simply connected, there is $\varphi \in H_{0}^{1}(\Omega)$ such that

$$
\mathbf{u}=\mathbf{w}+\nabla \varphi \quad \text { in } \quad \Omega .
$$

On $\Omega_{E}, \varphi$ satisfies

$$
\left\{\begin{array}{rlrl}
\Delta \varphi & =\operatorname{div} \mathbf{u} & & \text { in } \Omega_{E} \\
\varphi & =0 & & \text { on } \partial \Omega \\
\int_{B} \partial_{n} \varphi \mathrm{d} S & =\int_{B} \mathbf{w} \cdot \mathbf{n} \mathrm{d} S . &
\end{array}\right.
$$

According to Lemma 2.1, we have an estimate

$$
\|\varphi\|_{1, \Omega_{E}} \leq C\left(\|\operatorname{div} \mathbf{u}\|_{0, \Omega_{E}}+\left\|\left.\varphi\right|_{B}\right\|_{H^{1 / 2}(B) / \mathbb{C}}+\left|\int_{B} \mathbf{w} \cdot \mathbf{n} \mathrm{d} S\right|\right) .
$$

Because of

$$
\|\mathbf{u}\|_{0, \Omega_{E}} \leq\|\mathbf{w}\|_{0, \Omega_{E}}+\|\nabla \varphi\|_{0, \Omega_{E}} \lesssim\|\operatorname{curl} \mathbf{u}\|_{0, \Omega_{E}}+\|\varphi\|_{1, \Omega_{E}},
$$

it remains to bound $\left\|\left.\varphi\right|_{B}\right\|_{H^{1 / 2}(B) / \mathbb{C}}$ and $\left|\int_{B} \mathbf{w} \cdot \mathbf{n} \mathrm{d} S\right|$.

The latter clearly satisfies $\left|\int_{B} \mathbf{w} \cdot \mathbf{n} \mathrm{d} S\right| \lesssim\|\mathbf{w}\|_{1, \Omega} \lesssim\|\mathbf{c u r l} \mathbf{u}\|_{0, \Omega}$.

Finally (see $[20]$ for the trace estimates)

$$
\begin{aligned}
\left\|\left.\varphi\right|_{B}\right\|_{H^{1 / 2}(B) / \mathbb{C}} & \leq\|\mathbf{n} \times \nabla \varphi\|_{-1 / 2, B} \leq\|\mathbf{n} \times \mathbf{u}\|_{-1 / 2, B}+\|\mathbf{n} \times \mathbf{w}\|_{-1 / 2, B} \\
& \lesssim\|\mathbf{u}\|_{\mathbf{H}\left(\operatorname{curl}, \Omega_{C}\right)}+\|\mathbf{w}\|_{1, \Omega} \\
& \lesssim\|\mathbf{u}\|_{0, \Omega_{C}}+\|\mathbf{c u r l} \mathbf{u}\|_{0, \Omega_{C}}+\|\mathbf{c u r l} \mathbf{u}\|_{0, \Omega}
\end{aligned}
$$

\subsection{Variational problems}

For all $\delta \in\left[0, \delta_{0}\right]$, we consider the variational problem:

$$
\text { Find } \mathbf{E} \in \mathbf{Y}(\Omega) \text { s. t. } a_{R}^{\delta}(\mathbf{E}, \mathbf{v})=-i \omega\left(\mathbf{j}_{0}, \mathbf{v}\right)_{\Omega_{C}}, \quad \forall \mathbf{v} \in \mathbf{Y}(\Omega),
$$

where $(\cdot, \cdot)_{D}$ is the $L^{2}(D)^{3}$ hermitian inner product.

Theorem 2.3. Let $\mathbf{j}_{0}$ satisfy

$$
\mathbf{j}_{0} \in L^{2}(\Omega), \mathbf{j}_{0}=0 \text { in } \Omega_{E}, \operatorname{div} \mathbf{j}_{0}=0 \text { in } \Omega_{C}, \mathbf{j}_{0} \cdot \mathbf{n}=0 \text { on } B
$$

Let the positive constants $\mu_{C}, \mu_{E}, \hat{\varepsilon}_{C}, \hat{\varepsilon}_{E}, \sigma_{C}$ and $\omega$ be fixed. With $\delta_{0}$ given in Lemma 2.2 , for all $\delta \in\left[0, \delta_{0}\right]$ :

(i) there exists a unique solution $\mathbf{E}^{\delta}$ to problem (21);

(ii) the solution $\mathbf{E}^{\delta}$ satisfies all equations in (16);

(iii) the norms of the $\mathbf{E}^{\delta}$ in $\mathbf{Y}(\Omega)$ are uniformly bounded:

$$
\exists C>0, \quad \forall \delta \in\left[0, \delta_{0}\right], \quad\left\|\mathbf{E}^{\delta}\right\|_{\mathbf{Y}(\Omega)} \leq C ;
$$

(iv) as $\delta \rightarrow 0, \mathbf{E}^{\delta} \rightarrow \mathbf{E}^{0}$ and we have the convergence estimate

$$
\exists C>0, \quad \forall \delta \in\left[0, \delta_{0}\right], \quad\left\|\mathbf{E}^{\delta}-\mathbf{E}^{0}\right\|_{\mathbf{Y}(\Omega)} \leq C \delta .
$$


Proof. (i) is a mere consequence of Lemma 2.2 .

(ii) We first take as test functions $\mathbf{v}=\nabla \varphi$, with $\varphi_{C} \in H_{0}^{1}\left(\Omega_{C}, \Delta\right){ }^{(\dagger)}$ extended by zero outside $\Omega_{C}$. This yields

$$
\int_{\Omega_{C}}\left(\left(i \omega \sigma-\delta \omega^{2} \hat{\varepsilon}\right) \mathbf{E} \cdot \nabla \varphi+\operatorname{div} \mathbf{E} \operatorname{div} \nabla \varphi\right) \mathrm{d} x=-i \omega \int_{\Omega_{C}} \mathbf{j}_{0} \cdot \nabla \varphi \mathrm{d} x
$$

By Green's formula and the properties of $\mathbf{j}_{0}$, we obtain

$$
\int_{\Omega_{C}} \operatorname{div} \mathbf{E}\left(\left(-i \omega \sigma+\delta \omega^{2} \hat{\varepsilon}\right) \varphi+\Delta \varphi\right) \mathrm{d} x=0, \quad \forall \varphi \in H_{0}^{1}\left(\Omega_{C}, \Delta\right) .
$$

This yields (16) (ii) since $\left(-i \omega \sigma+\delta \omega^{2} \hat{\varepsilon}\right) \varphi+\Delta \varphi$ runs through the whole $L^{2}\left(\Omega_{C}\right)$ for $\varphi \in H_{0}^{1}\left(\Omega_{C}, \Delta\right)$.

A similar argument in $\Omega_{E}$ yields (16) (iv) since, as a consequence of Lemma 2.1, for $\delta$ small enough, the operator $\varphi \mapsto \delta \omega^{2} \hat{\varepsilon} \varphi+\Delta \varphi$ is surjective from

$$
\left\{\varphi \in H^{1}\left(\Omega_{E}\right):\left.\varphi\right|_{B}=c,\left.\varphi\right|_{\partial \Omega}=0, \int_{B} \partial_{n} \varphi \mathrm{d} S=0, \Delta \varphi \in L^{2}\left(\Omega_{E}\right)\right\}
$$

onto $L^{2}\left(\Omega_{E}\right)$.

Next for any $\chi \in H^{1 / 2}(B)$, we take $\mathbf{v}=\nabla \varphi$ with $\varphi$ in the space (17) such that $\varphi_{E}$ is solution of the Dirichlet problem $\Delta \varphi_{E}=0$ in $\Omega_{E}$ and $\varphi_{E}=\chi+c$ on $B$ (we use once more Lem. 2.1). Using this test function in (21), we get

Hence

$$
\int_{\Omega_{C}}(i \delta \omega \hat{\varepsilon} \mathbf{E}+\sigma \mathbf{E}) \cdot \nabla \varphi \mathrm{d} x+\int_{\Omega_{E}} i \delta \omega \hat{\varepsilon} \mathbf{E} \cdot \nabla \varphi \mathrm{d} x=-\int_{\Omega_{C}} \mathbf{j}_{0} \cdot \nabla \varphi \mathrm{d} x
$$

$$
\int_{B}(i \delta \omega[\hat{\varepsilon} \mathbf{E}]+\sigma \mathbf{E}) \cdot \mathbf{n} \chi \mathrm{d} S-c \int_{B} i \delta \omega \hat{\varepsilon}_{E} \mathbf{E}_{E} \cdot \mathbf{n} \mathrm{d} S=0 .
$$

Since $\int_{B} \mathbf{E}_{E} \cdot \mathbf{n}=0$, we conclude that we have (16) (vii).

The other equations of (16) are then obtained in a standard way.

(iii) is a consequence of the uniform coerciveness proved in Lemma 2.2.

(iv) We have for all $\mathbf{v} \in \mathbf{Y}(\Omega)$ :

$$
a_{R}^{0}\left(\mathbf{E}^{\delta}-\mathbf{E}^{0}, \mathbf{v}\right)=a_{R}^{0}\left(\mathbf{E}^{\delta}, \mathbf{v}\right)-a^{\delta}\left(\mathbf{E}^{\delta}, \mathbf{v}\right)=\delta \int_{\Omega} \omega^{2} \hat{\varepsilon} \mathbf{E}^{\delta} \cdot \overline{\mathbf{v}} \mathrm{d} x .
$$

Taking $\mathbf{v}=\mathbf{E}^{\delta}-\mathbf{E}^{0}$ and using the uniform coerciveness estimate, we obtain

$$
\left\|\mathbf{E}^{\delta}-\mathbf{E}^{0}\right\|_{\mathbf{Y}(\Omega)}^{2} \leq C \delta\left\|\mathbf{E}^{\delta}\right\|_{L^{2}(\Omega)}\left\|\mathbf{E}^{\delta}-\mathbf{E}^{0}\right\|_{L^{2}(\Omega)}
$$

With the help of the continuous imbeddings $\mathbf{Y}(\Omega) \subset L^{2}(\Omega)^{3} \subset \mathbf{Y}^{\prime}(\Omega)$ we conclude, thanks to (iii).

Remark 2.1. All results above extend to the case when $B$ is not simply connected. Let $B_{i}$ for $i=1, \ldots, I$ be the connected components of $B$. Let us prove that

$$
(\mathbf{E}, \mathbf{H}) \text { solution of }(1)-(2) \quad \Longrightarrow \int_{B_{i}} \mathbf{E}_{E} \cdot \mathbf{n} \mathrm{d} S=0, i=1, \ldots, I \text {. }
$$

\footnotetext{
${ }^{(\dagger)}$ Here $H_{0}^{1}\left(\Omega_{C}, \Delta\right)$ is the subspace of the $\varphi \in H_{0}^{1}\left(\Omega_{C}\right)$ such that $\Delta \varphi_{C} \in L^{2}\left(\Omega_{C}\right)$.
} 
The equation $\operatorname{div} \mathbf{E}_{C}=0$ is not sufficient now to deduce (23). By [4, Lem. 3.5] we know that there exists a vector potential $\mathbf{J}_{0} \in H^{1}\left(\mathbf{c u r l}, \Omega_{C}\right)$ for $\mathbf{j}_{0}: \mathbf{j}_{0}=\mathbf{c u r l} \mathbf{J}_{0}$ in $\Omega_{C}$. Therefore equation (2) yields that

$$
\mathbf{E}=\operatorname{curl} \boldsymbol{\psi} \text { with } \quad \boldsymbol{\psi}=\left(i \omega \varepsilon_{C}+\sigma_{C}\right)^{-1}\left(\mathbf{H}-\mathbf{J}_{0}\right)
$$

The proof goes as in [4, Lem. 3.5]: Let $\mu_{i} \in \mathcal{C}_{0}^{\infty}\left(\mathbb{R}^{3}\right)$ such that $\mu_{i} \equiv 1$ in a neighborhood of $B_{i}$ and $\mu_{i} \equiv 0$ in a neighborhood of the other connected components of $B$. Then

$$
\int_{B_{i}} \mathbf{E}_{C} \cdot \mathbf{n} \mathrm{d} S=\int_{\Omega_{C}} \operatorname{div}\left\{\operatorname{curl}\left(\mu_{i} \boldsymbol{\psi}\right)\right\} \mathrm{d} x=0
$$

Then we deduce that $\int_{B_{i}} \mathbf{E}_{E} \cdot \mathbf{n} \mathrm{d} S=0$ as before. The gauge conditions for the eddy current problem are now $\operatorname{div} \mathbf{E}_{E}=0$ in $\Omega_{E}$ and

$$
\int_{B_{i}} \mathbf{E}_{E} \cdot \mathbf{n} \mathrm{d} S=0, \quad i=1, \ldots, I
$$

In the definition of the space $\mathbf{Y}(\Omega)$ the gauge conditions $(24)$ are now present.

The modification of Lemma 2.1 is obvious: The boundary conditions on $B_{i}$ are $\varphi=v+c_{i}$ and $\int_{B_{i}} \partial_{n} \varphi \mathrm{d} S=b_{i}$ with $b_{i}$ given constants. The estimate (19) contains the term $\sum_{i}\left|b_{i}\right|$ instead of $|b|$. The proof relies on the full [4, Prop. 3.18]. Lemma 2.2 and Theorem 2.3 are still valid under these assumptions. The extension of the proofs is straightforward.

\section{Singularities of the Variational spaCes}

In this section, we investigate the splitting of the variational solutions of (21) into the sum of a regular field $\mathbf{w} \in H^{1}(\Omega)^{3}$ and of a singular gradient $\nabla \Phi$, where $\Phi$ is not, in general, in $H^{2}(\Omega)$.

\subsection{General situation}

The space $\mathbf{Y}(\Omega)$ contains some of the essential boundary conditions appearing in (16), namely, (16) (vi) and (viii). But the essential condition (16) (vii) depends on $\delta$. On the other hand we do not impose the gauge condition $\int_{B} \mathbf{E}_{E} \cdot \mathbf{n} \mathrm{d} S=0$ for this analysis. Let us then set

$$
\mathbf{X}(\Omega)=\left\{\mathbf{u} \in \mathbf{H}_{0}(\mathbf{c u r l}, \Omega): \operatorname{div} \mathbf{u}_{C} \in L^{2}\left(\Omega_{C}\right), \operatorname{div} \mathbf{u}_{E} \in L^{2}\left(\Omega_{E}\right)\right\}
$$

and for $\delta \geq 0$ :

$$
\mathbf{X}^{\delta}(\Omega)=\left\{\mathbf{u} \in \mathbf{X}(\Omega): i \delta \omega[\hat{\varepsilon} \mathbf{u} \cdot \mathbf{n}]+\sigma_{C} \mathbf{u}_{C} \cdot \mathbf{n}=0 \text { on } B\right\}
$$

In fact, the solution of $(21)$ belongs to $\mathbf{X}^{\delta}(\Omega)$. Note that the variational formulation could equivalently be set in $\mathbf{X}^{\delta} \cap \mathbf{Y}(\Omega)$, but, in order to prove the convergence result as $\delta \rightarrow 0$ we preferred to use a space independent of $\delta$.

Let us recall more classical notations [15]: For a domain $D$

$$
\mathbf{X}_{N}(D)=\left\{\mathbf{u} \in \mathbf{H}_{0}(\operatorname{curl}, D): \operatorname{div} \mathbf{u} \in L^{2}(D)\right\}
$$

and

$$
\mathbf{X}_{T}(D)=\left\{\mathbf{u} \in \mathbf{H}(\mathbf{c u r l}, D): \operatorname{div} \mathbf{u} \in L^{2}(D), \mathbf{u} \cdot \mathbf{n}=0 \text { on } \partial D\right\}
$$

By a straightforward adaptation of the result [17, Th. 3.5] to the situation of complex coefficients, we obtain the splitting result for the spaces $\mathbf{X}^{\delta}(\Omega)$ when $\delta>0$. In order to state it, we need the introduction of the interface Laplacian $\Delta_{\underline{\delta}}$ over $H_{0}^{1}(\Omega)$ :

$$
\left(\Delta_{\underline{\delta}} \varphi, \psi\right)_{\Omega}=\int_{\Omega_{C}}\left(\sigma_{C}+i \delta \omega \hat{\varepsilon}_{C}\right) \nabla \varphi_{C} \cdot \nabla \bar{\psi}_{C}+\int_{\Omega_{E}}\left(i \delta \omega \hat{\varepsilon}_{E}\right) \nabla \varphi_{E} \cdot \nabla \bar{\psi}_{E},
$$


for any $\varphi, \psi \in H_{0}^{1}(\Omega)$. Then for $\delta>0$, under a technical condition ${ }^{(\ddagger)}$, any field $\mathbf{v} \in \mathbf{X}^{\delta}(\Omega)$ admits a decomposition

$$
\mathbf{v}=\mathbf{w}+\nabla \Phi
$$

where $\mathbf{w} \in \mathbf{X}^{\delta}(\Omega)$ is such that $\mathbf{w}_{C} \in H^{1}\left(\Omega_{C}\right)^{3}, \mathbf{w}_{E} \in H^{1}\left(\Omega_{E}\right)^{3}$ and $\Phi \in H_{0}^{1}(\Omega)$ satisfies $\Delta_{\underline{\delta}} \Phi \in L^{2}(\Omega)$.

\subsection{The eddy current case}

The goal of this subsection is to describe the decomposition of vector fields from the eddy current variational space $\mathbf{X}^{0}(\Omega)$ into regular fields and singular ones in the spirit of $[5,7-9,15,17]$ (and even using some results from these papers).

Theorem 3.1. Any field $\mathbf{v} \in \mathbf{X}^{0}(\Omega)$ admits a decomposition

$$
\mathbf{v}=\mathbf{w}+\nabla \Phi
$$

with $\mathbf{w} \in \mathbf{X}^{0}(\Omega)$ such that $\mathbf{w}_{C} \in H^{1}\left(\Omega_{C}\right)^{3}, \mathbf{w}_{E} \in H^{1}\left(\Omega_{E}\right)^{3}$ and the potential $\Phi \in H^{1}(\Omega)$ satisfies

$$
\begin{aligned}
& \Delta \Phi_{C} \in L^{2}\left(\Omega_{C}\right), \\
& \partial_{n} \Phi_{C}=0 \quad \text { on } B, \\
& \Delta \Phi_{E} \in L^{2}\left(\Omega_{E}\right), \\
& \Phi_{E}=0 \quad \text { on } \partial \Omega .
\end{aligned}
$$

Proof. We remark that the restriction $\mathbf{v}_{C}$ of $\mathbf{v}$ to $\Omega_{C}$ belongs to $\mathbf{X}_{T}\left(\Omega_{C}\right)$. Therefore by Theorem 1.1 of [15] (see also $[7,8]$ or Th. 3.5 of $[17]$ ), $\mathbf{v}_{C}$ admits a decomposition

$$
\mathbf{v}_{C}=\mathbf{w}_{C}+\nabla \Phi_{C} \text { in } \Omega_{C},
$$

where $\mathbf{w}_{C} \in H^{1}\left(\Omega_{C}\right)^{3} \cap \mathbf{X}_{T}\left(\Omega_{C}\right)$ and $\Phi_{C} \in H^{1}\left(\Omega_{C}\right)$ satisfies (28)-(29).

Now consider $\chi \in H^{1}\left(\Omega_{E}\right)$ the unique weak solution of

$$
\begin{array}{rlrl}
\Delta \chi & =0 & \text { in } \Omega_{E}, \\
\chi & =\Phi_{C} & & \text { on } B .
\end{array}
$$

Denote by $\widetilde{\Phi}_{C}$ the function defined by

$$
\tilde{\Phi}_{C}=\left\{\begin{array}{lll}
\Phi_{C} & \text { in } & \Omega_{C} \\
\chi & \text { in } & \Omega_{E}
\end{array}\right.
$$

By construction $\widetilde{\Phi}_{C}$ belongs to $H^{1}(\Omega)$. Denote furthermore by $\widetilde{\mathbf{w}}_{C}$ an extension of $\mathbf{w}_{C}$ to $\Omega$ which belongs to $H^{1}(\Omega)^{3}$ and is zero on $\partial \Omega$. Let us now set

$$
\widetilde{\mathbf{v}}_{C}=\widetilde{\mathbf{w}}_{C}+\nabla \widetilde{\Phi}_{C}
$$

Then by construction this is equal to $\mathbf{v}_{C}$ in $\Omega_{C}$ and it satisfies

$$
\left[\widetilde{\mathbf{v}}_{C} \times \mathbf{n}\right]=0 \text { on } B
$$

These properties imply that $\mathbf{u}_{E}$ defined in $\Omega_{E}$ by

$$
\mathbf{u}_{E}=\mathbf{v}_{E}-\left.\widetilde{\mathbf{v}}_{C}\right|_{\Omega_{E}}
$$

${ }^{(\ddagger)}$ The interface Laplacian $\Delta_{\underline{\delta}}$ has no edge exponent equal to 1 and no corner exponent equal to $\frac{1}{2}$. This condition is probably not necessary. 
satisfies

$$
\begin{aligned}
& \mathbf{u}_{E} \times \mathbf{n}=0 \text { on } B \\
& \text { curl } \mathbf{u}_{E}=\operatorname{curl} \mathbf{v}_{E}-\left.\operatorname{curl} \widetilde{\mathbf{w}}_{C}\right|_{\Omega_{E}} \in L^{2}\left(\Omega_{E}\right)^{3}, \\
& \operatorname{div} \mathbf{u}_{E}=\operatorname{div} \mathbf{v}_{E}-\left.\operatorname{div} \widetilde{\mathbf{w}}_{C}\right|_{\Omega_{E}}-\operatorname{div}(\nabla \chi) \in L^{2}\left(\Omega_{E}\right) .
\end{aligned}
$$

This means that $\mathbf{u}_{E}$ belongs to $\mathbf{X}_{N}\left(\Omega_{E}\right)$. Again by Theorem 1.1 of [15] (see also [7,8] or Th. 3.5 of [17]) $\mathbf{u}_{E}$ admits a decomposition

$$
\mathbf{u}_{E}=\mathbf{w}_{R E}+\nabla \varphi_{E} \text { in } \Omega_{E},
$$

where $\mathbf{w}_{R E} \in H^{1}\left(\Omega_{E}\right)^{3} \cap \mathbf{X}_{N}\left(\Omega_{E}\right)$ and $\varphi_{E}$ belongs to $H^{1}\left(\Omega_{E}\right)$ and satisfies (30) and the Dirichlet boundary condition

$$
\varphi_{E}=0 \text { on } B \cup \partial \Omega .
$$

This decomposition (35) into the splitting (34) gives with the help of (33)

$$
\mathbf{v}_{E}=\mathbf{w}_{R E}+\nabla \varphi_{E}+\left.\widetilde{\mathbf{w}}_{C}\right|_{\Omega_{E}}+\nabla \chi \quad \text { in } \quad \Omega_{E}
$$

or equivalently

$$
\mathbf{v}_{E}=\mathbf{w}_{E}+\nabla \Phi_{E} \quad \text { in } \Omega_{E},
$$

once we set $\mathbf{w}_{E}=\mathbf{w}_{R E}+\left.\widetilde{\mathbf{w}}_{C}\right|_{\Omega_{E}}$ and $\Phi_{E}=\varphi_{E}+\chi$. The conclusion follows from (32), (36) and the above properties of $\mathbf{w}_{R E}, \widetilde{\mathbf{w}}_{C}, \phi_{E}$ and $\chi$.

The relation between the general decomposition (26) and Theorem 3.1 in the limit when $\delta \rightarrow 0$ is not straightforward and will be clarified later.

\section{LAPlaCe Singularities FOR THE POTENTIALS}

The singularities of the Maxwell and eddy current problems are produced by the corners a and the edges e of $\Omega_{C}$, - Note that the corners and edges are all part of the interface $B$. Concerning the Maxwell interface problems (corresponding to $\delta>0$ ), these singularities are known [17] to derive from those of scalar problems for potentials, namely $\Delta_{\underline{\delta}}$ and $\Delta_{\mu}$, where $\Delta_{\underline{\delta}}$ is defined in (25) and the latter operator is defined as:

$$
\left(\Delta_{\mu} \varphi, \psi\right)_{\Omega}=\int_{\Omega_{C}} \mu_{C} \nabla \varphi_{C} \cdot \nabla \bar{\psi}_{C}+\int_{\Omega_{E}} \mu_{E} \nabla \varphi_{E} \cdot \nabla \bar{\psi}_{E},
$$

for any $\varphi, \psi \in H^{1}(\Omega)$.

We will now recall the singularities of these two interface Laplacians $\Delta_{\underline{\delta}}$ (electric) and $\Delta_{\mu}$ (magnetic). For the sake of brevity we restrict ourselves to a minimal description and refer to $[15,17,24,26-28]$ for more details. Moreover, we describe the singularities of the coupled Neumann-Dirichlet problem (28)-(31) of the eddy current problem. We show that their exponents (i.e. degrees of homogeneity) are the limit of the interface singularity exponents of $\Delta_{\underline{\delta}}$ as $\delta \rightarrow 0$. We give complements on the behavior of all singularities (scalar and Maxwell) in the eddy current limit as $\delta \rightarrow 0$ in Section 7 .

\subsection{General definitions for Laplace singularities in cones and sectors}

As we know from [23], the singularities (singular parts of solutions) of elliptic problems at a corner 0 are obtained as non-zero quasi-homogeneous solutions of the same problem with zero right hand side in the infinite cone (or sector) which coincides with the finite domain at this corner 0 . 
Let $\Gamma$ be an infinite cone in $\mathbb{R}^{d}$ for $d=3$ or 2 ( $\Gamma$ is then a sector), centered in 0 . Let $(\rho, \vartheta)$ be the polar coordinates centered at 0 . Let $G$ be the intersection of $\Gamma$ with the unit sphere. The singularities in $\Gamma$ are quasi-homogeneous functions: Let us set for $\lambda \in \mathbb{C}$ :

$$
S^{\lambda}(\Gamma)=\left\{\Psi(x)=\rho^{\lambda} \sum_{q=0}^{Q}(\log \rho)^{q} \psi_{q}(\vartheta): \psi_{q} \in H^{1}(G)\right\}
$$

The singularities of an elliptic problem are the non-zero solutions in some $S^{\lambda}(\Gamma)$ of the same problem with zero right hand side. The corresponding $\lambda$ are the exponents of singularities.

The set of exponents can be found by searching solutions in the subspace of homogeneous functions, $\bar{S}^{\lambda}(\Gamma):=$ $\left\{\Psi(x)=\rho^{\lambda} \psi(\vartheta): \psi \in H^{1}(G)\right\}$.

\subsubsection{Dirichlet problem.}

We denote the set of exponents of the Dirichlet problem for $\Delta$ on $\Gamma$ by $\Lambda^{\operatorname{Dir}}(\Gamma)$, i.e. the $\lambda$ for which there exists a non-zero $\Psi \in \bar{S}^{\lambda}(\Gamma)$, solution of the problem

$$
\Delta \Psi=0 \text { in } \Gamma \text { and } \Psi=0 \text { on } \partial \Gamma \text {. }
$$

For $\lambda$ in this set, let $Z_{\text {Dir }}^{\lambda}(\Gamma)$ be the corresponding space of singularities.

Let $L$ be the positive Laplace-Beltrami operator on the unit sphere $\left(L=-\partial_{\vartheta}^{2}\right.$ if $\left.d=2\right)$. For $\Psi(x)=\rho^{\lambda} \psi(\vartheta)$, we have $-\Delta \Psi=\rho^{\lambda-2}(L \psi-\nu \psi)$ where

$$
\nu=\lambda^{2} \text { if } d=2 \text { and } \quad \nu=\lambda(\lambda+1) \text { if } d=3 .
$$

Thus it is standard to prove that $\Lambda^{\operatorname{Dir}}(\Gamma)$ is the set $\lambda$ such that $\nu$ in $(38)$ is an eigenvalue of the Dirichlet problem for $L$ on $G$. Moreover $Z_{\text {Dir }}^{\lambda}(\Gamma)$ is the space of $\rho^{\lambda} \psi(\vartheta)$ with $\psi$ an eigenvector associated with the eigenvalue $\nu$ in $(38)$.

\subsubsection{Neumann problem.}

The set $\Lambda^{\mathrm{Neu}}(\Gamma)$ of Neumann exponents is similarly defined as the $\lambda$ for which there exists a non-zero $\Psi \in \bar{S}^{\lambda}(\Gamma)$, solution of

$$
\Delta \Psi=0 \text { in } \Gamma \text { and } \partial_{n} \Psi=0 \text { on } \partial \Gamma .
$$

The space $Z_{\text {Neu }}^{\lambda}(\Gamma)$ is defined analogously and the Neumann eigenpairs of $L$ on $G$ yield the singularities as above.

\subsubsection{Interface problems.}

The interface problems that we consider in most of this paper are of simple type. They correspond to the separation of the whole space $\Gamma=\mathbb{R}^{2}$ or $\mathbb{R}^{3}$ into two conical regions $\Gamma_{C}$ and $\Gamma_{E}$, i.e. $\Gamma=\bar{\Gamma}_{C} \cup \bar{\Gamma}_{E}$ and $\Gamma_{C} \cap \Gamma_{E}=\emptyset$. We note that

$$
S^{\lambda}(\Gamma)=\left\{\Psi: \Psi_{C} \in S^{\lambda}\left(\Gamma_{C}\right), \quad \Psi_{E} \in S^{\lambda}\left(\Gamma_{E}\right), \quad \Psi_{C}=\Psi_{E} \text { on } I:=\partial \Gamma_{C}=\partial \Gamma_{E}\right\}
$$

Let $\alpha$ be a piecewise constant function, equal to $\alpha_{C} \in \mathbb{C}$ in $\Gamma_{C}$ and to $\alpha_{E} \in \mathbb{C}$ in $\Gamma_{E}$. The set of exponents of the interface problem associated with the operator

$$
(\Phi, \Psi) \longmapsto \int_{\Gamma_{C}} \alpha_{C} \nabla \Phi_{C} \cdot \nabla \bar{\Psi}_{C}+\int_{\Gamma_{E}} \alpha_{E} \nabla \Phi_{E} \cdot \nabla \bar{\Psi}_{E}
$$


is the set $\Lambda_{\alpha}(\Gamma)$ of the $\lambda$ for which there exists a non-zero $\Psi \in \bar{S}^{\lambda}(\Gamma)$, solution of (see, e.g., $[19,24,25,29]$ )

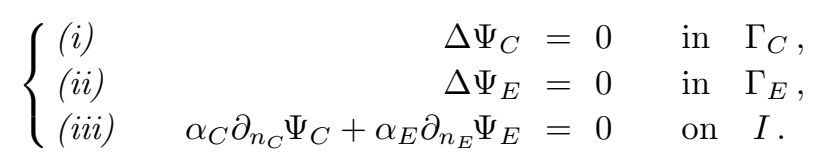

For $\lambda$ in this set, let $Z^{\lambda}(\Gamma ; \alpha)$ be the corresponding space of singularities. Then the $\lambda \in \Lambda_{\alpha}(\Gamma)$ are such that $\nu$ in (38) are the eigenvalues of the problem

$$
\psi \in H^{1}\left(\mathbb{S}^{d-1}\right), \quad \forall \varphi \in H^{1}\left(\mathbb{S}^{d-1}\right), \int_{\mathbb{S}^{d-1}} \alpha \nabla \psi \cdot \nabla \varphi=\nu \int_{\mathbb{S}^{d-1}} \alpha \psi \varphi
$$

When $\alpha>0$, the space $Z^{\lambda}(\Gamma ; \alpha)$ contains only homogeneous functions of the form $\rho^{\lambda} \psi(\vartheta)$ with $\psi$ solution of (42).

\subsection{The eddy current limit for potentials}

For $\delta>0$, the singularities of the electric transmission Laplacian $\Delta_{\underline{\delta}}$ are the non-zero $\Psi \in \bar{S}^{\lambda}(\Gamma)$ solution of the problem (41) with $\alpha=\alpha^{\delta}$ where

$$
\alpha_{C}^{\delta}=\sigma_{C}+i \delta \omega \hat{\varepsilon}_{C} \quad \text { and } \quad \alpha_{E}^{\delta}=i \delta \omega \hat{\varepsilon}_{E} .
$$

Going back to problem (28)-(31) for the eddy current potentials, we see that its singularities are the solutions $\Psi \in \bar{S}^{\lambda}(\Gamma)$ of the problem (41) with $\alpha=\alpha^{0}$, where $\alpha_{C}^{0}=\sigma_{C}$ and $\alpha_{E}^{0}=0$. Inserting $\Psi(x)=\rho^{\lambda} \psi(\vartheta)$ in (41) with $\alpha=\alpha^{0}$ we obtain the eigenvalue problem:

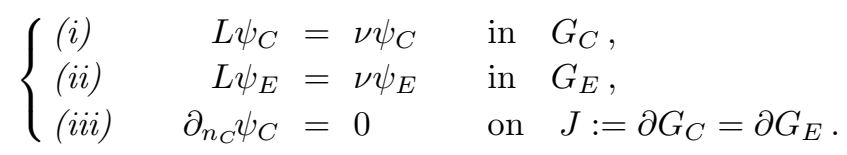

We are going to exhibit a common variational formulation for problems (42) and (44), and deduce that the spectrum of (42) for $\alpha=\alpha^{\delta}$ tends to the spectrum of (44) as $\delta \rightarrow 0$.

Let us divide equation (42) for $\alpha=\alpha^{\delta}$ by $\sigma_{C}+i \delta \omega \hat{\varepsilon}_{C}$ and let us set

$$
\eta=\frac{i \delta \omega \hat{\varepsilon}_{E}}{\sigma_{C}+i \delta \omega \hat{\varepsilon}_{C}} .
$$

Then the eigenvalue problem (42) with $\alpha=\alpha^{\delta}$ becomes

Find $\psi \in H^{1}\left(\mathbb{S}^{d-1}\right), \psi \neq 0, \forall \varphi \in H^{1}\left(\mathbb{S}^{d-1}\right)$ :

$$
\int_{G_{C}} \nabla \psi_{C} \cdot \nabla \varphi_{C}+\eta \int_{G_{E}} \nabla \psi_{E} \cdot \nabla \varphi_{E}=\nu\left\{\int_{G_{C}} \psi_{C} \varphi_{C}+\eta \int_{G_{E}} \psi_{E} \varphi_{E}\right\} .
$$

Let us denote by $P_{E}$ the harmonic extension from $G_{C}$ into $G_{E}$ : For $\psi_{C}$ in $H^{1}\left(G_{C}\right), P_{E} \psi_{C}$ is the solution of $L \varphi=0$ in $G_{E}$ and $\varphi=\psi_{C}$ on $\partial G_{E}=\partial G_{C}$. Consequently there holds

$$
\forall \varphi_{0} \in H_{0}^{1}\left(G_{E}\right), \quad \int_{G_{E}} \nabla\left(P_{E} \psi_{C}\right) \cdot \nabla \varphi_{0}=0 .
$$

For any $\psi \in H^{1}\left(\mathbb{S}^{d-1}\right)$, we have

$$
\psi_{E}=P_{E} \psi_{C}+\psi_{0}, \quad \text { where } \quad \psi_{0} \in H_{0}^{1}\left(G_{E}\right) .
$$


We write similarly $\varphi_{E}=P_{E} \varphi_{C}+\varphi_{0}$, with $\varphi_{0} \in H_{0}^{1}\left(G_{E}\right)$. Inserting this in (46) and using (47), we obtain $\forall \varphi_{C} \in H^{1}\left(G_{C}\right)$ and $\forall \varphi_{0} \in H_{0}^{1}\left(G_{E}\right)$ :

$$
\begin{aligned}
\int_{G_{C}} \nabla \psi_{C} \cdot \nabla \varphi_{C}+\eta \int_{G_{E}}\left(\nabla P_{E} \psi_{C} \cdot \nabla P_{E} \varphi_{C}+\nabla \psi_{0} \cdot \nabla \varphi_{0}\right)= \\
\quad \nu\left\{\int_{G_{C}} \psi_{C} \varphi_{C}+\eta \int_{G_{E}}\left(P_{E} \psi_{C} P_{E} \varphi_{C}+P_{E} \psi_{C} \varphi_{0}+\psi_{0} P_{E} \varphi_{C}+\psi_{0} \varphi_{0}\right)\right\},
\end{aligned}
$$

i.e.

$$
\begin{aligned}
\forall \varphi_{C} \in H^{1}\left(G_{C}\right), \int_{G_{C}} \nabla \psi_{C} \cdot \nabla \varphi_{C}+\eta \int_{G_{E}} \nabla P_{E} \psi_{C} \cdot \nabla P_{E} \varphi_{C}= \\
\nu\left\{\int_{G_{C}} \psi_{C} \varphi_{C}+\eta \int_{G_{E}}\left(P_{E} \psi_{C} P_{E} \varphi_{C}+\psi_{0} P_{E} \varphi_{C}\right)\right\}
\end{aligned}
$$

and

$$
\forall \varphi_{0} \in H_{0}^{1}\left(G_{E}\right), \quad \int_{G_{E}} \nabla \psi_{0} \cdot \nabla \varphi_{0}=\nu \int_{G_{E}}\left(P_{E} \psi_{C} \varphi_{0}+\psi_{0} \varphi_{0}\right) .
$$

For $\eta=0$, equation (48) becomes

$$
\forall \varphi_{C} \in H^{1}\left(G_{C}\right), \quad \int_{G_{C}} \nabla \psi_{C} \cdot \nabla \varphi_{C}=\nu \int_{G_{C}} \psi_{C} \varphi_{C}
$$

The solutions of the system (49)-(50) are the solutions of system (44). Thus we have written the eigenproblems (42) for $\alpha=\alpha^{\delta}$ and (44) using the unified variational formulation (48)-(49), which has the form Find $\left(\psi_{C}, \psi_{0}\right) \in H^{1}\left(G_{C}\right) \times H_{0}^{1}\left(G_{E}\right), \forall\left(\varphi_{C}, \varphi_{0}\right) \in H^{1}\left(G_{C}\right) \times H_{0}^{1}\left(G_{E}\right)$ :

$$
a_{\eta}\left(\psi_{C}, \psi_{0} ; \varphi_{C}, \varphi_{0}\right)=\nu b_{\eta}\left(\psi_{C}, \psi_{0} ; \varphi_{C}, \varphi_{0}\right)
$$

where $a_{\eta}$ and $b_{\eta}$ depend continuously on $\eta \in\left[0, \eta_{0}\right]$.

As a consequence of the analysis above, we have proved the following:

\section{Proposition 4.1.}

(i) For $\eta=0$, the set of eigenvalues $\nu$ of the system (49)-(50) is the union of the set of Neumann eigenvalues in $G_{C}$ and the set of Dirichlet eigenvalues in $G_{E}$.

(ii) When $\eta \rightarrow 0$, the eigenvalues of the system (48)-(49) tend to the eigenvalues of the system (49)-(50).

\subsection{Corner singularities}

Now, we go back to the specific description of the singularities of the interface Laplacians $\Delta_{\underline{\delta}}$ (and problem (28)-(31) for $\delta=0)$ and $\Delta_{\mu}$ at the corners of $\Omega_{C}$. Note that the external Dirichlet or Neumann boundary conditions hold on the external smooth boundary $\partial \Omega$ and do not influence the interface singularities.

Fix a corner $\mathbf{a} \in B$. There exist infinite polyhedral cones $\Gamma_{C, \mathbf{a}}$ and $\Gamma_{E, \mathbf{a}}$ with vertex $\mathbf{a}$ and such that for $\rho_{0}>0$ small enough

$$
\Omega_{C} \cap \mathcal{B}\left(\mathbf{a}, \rho_{0}\right)=\Gamma_{C, \mathbf{a}} \cap \mathcal{B}\left(\mathbf{a}, \rho_{0}\right) \quad \text { and } \quad \Omega_{E} \cap \mathcal{B}\left(\mathbf{a}, \rho_{0}\right)=\Gamma_{E, \mathbf{a}} \cap \mathcal{B}\left(\mathbf{a}, \rho_{0}\right) .
$$

Note that $\bar{\Gamma}_{C, \mathbf{a}} \cup \bar{\Gamma}_{E, \mathbf{a}}$ is the full space $\Gamma=\mathbb{R}^{3}$. We refer to this conical partition of $\mathbb{R}^{3}$ associated with a by the notation $\Gamma_{\mathbf{a}}$.

Then we denote by $\Lambda_{\underline{\delta}}\left(\Gamma_{\mathbf{a}}\right)$ the set of the exponents determined by problem (41) with $\alpha=\alpha^{\delta}$, see (43), for $\delta \geq 0$, and with $\Gamma_{C}=\Gamma_{C}, \mathbf{a}$ and $\Gamma_{E}=\Gamma_{E, \mathbf{a}}$. We denote similarly the spaces of singularities by $Z^{\lambda}\left(\Gamma_{\mathbf{a}}, \underline{\delta}\right)$. As a consequence of Proposition 4.1, we obtain 
Lemma 4.2. For $\delta=0$, the set of singular exponents satisfies

$$
\Lambda_{\underline{0}}\left(\Gamma_{\mathbf{a}}\right)=\Lambda^{\mathrm{Neu}}\left(\Gamma_{C, \mathbf{a}}\right) \cup \Lambda^{\operatorname{Dir}}\left(\Gamma_{E, \mathbf{a}}\right) .
$$

When $\delta \rightarrow 0$, the set $\Lambda_{\underline{\delta}}\left(\Gamma_{\mathbf{a}}\right)$ tends to $\Lambda_{\underline{0}}\left(\Gamma_{\mathbf{a}}\right)$.

The exponents and singular spaces associated with $\Delta_{\mu}$ are denoted by $\Lambda_{\mu}\left(\Gamma_{\mathbf{a}}\right)$ and $Z^{\lambda}\left(\Gamma_{\mathbf{a}}, \mu\right)$, according to Section 4.2 (iii).

\subsection{Edge singularities}

Let $\mathbf{e} \subset B$ be an edge of $\partial \Omega_{C}$. There exist two plane sectors $\Gamma_{C, \mathbf{e}}$ and $\Gamma_{E, \mathbf{e}}$ such that for any point $x \in \mathbf{e}$ there exists a neighborhood $\mathcal{B}$ of $x$ and a Cartesian system of coordinates such that

$$
\Omega_{C} \cap \mathcal{B}=\left(\Gamma_{C, \mathbf{e}} \times \mathbb{R}\right) \cap \mathcal{B} \text { and } \quad \Omega_{E} \cap \mathcal{B}=\left(\Gamma_{E, \mathbf{e}} \times \mathbb{R}\right) \cap \mathcal{B}
$$

Let $\omega_{C, \mathbf{e}}$ and $\omega_{E, \mathbf{e}}$ be the opening of $\Gamma_{C, \mathbf{e}}$ and $\Gamma_{E, \mathbf{e}}$ respectively. Of course we have $\omega_{C, \mathbf{e}}+\omega_{E, \mathbf{e}}=2 \pi$. We refer to this partition of $\mathbb{R}^{2}$ into two sectors associated with $\mathbf{e}$ by the notation $\Gamma_{\mathbf{e}}$.

Like for corners, we denote by $\Lambda_{\underline{\delta}}\left(\Gamma_{\mathbf{e}}\right)$ the set of the exponents determined by problem (41) with $\alpha=\alpha^{\delta}$, see (43), for $\delta \geq 0$, and with $\Gamma_{C} \stackrel{=}{=} \Gamma_{C}$, e and $\Gamma_{E}=\Gamma_{E}$, e. We denote similarly the spaces of singularities by $Z^{\lambda}\left(\Gamma_{\mathbf{e}}, \underline{\delta}\right)$. As a consequence of Proposition 4.1 , we obtain

Lemma 4.3. For $\delta=0$, the set of singular exponents satisfies

$$
\Lambda_{\underline{0}}\left(\Gamma_{\mathbf{e}}\right)=\left\{\frac{k \pi}{\omega_{C, \mathbf{e}}}: k \in \mathbb{Z}\right\} \cup\left\{\frac{k \pi}{\omega_{E, \mathbf{e}}}: k \in \mathbb{Z} \backslash\{0\}\right\} .
$$

For $\delta>0, \Lambda_{\underline{\delta}}\left(\Gamma_{\mathbf{e}}\right)$ is the set of $\lambda=\sqrt{\nu}$ with $\nu$ solution in $\mathbb{C}$ of the equation

$$
(1+\eta) \sin \nu \pi= \pm(1-\eta) \sin \nu\left(\pi-\omega_{C, \mathbf{e}}\right), \quad \text { with } \eta \text { given in }(45)
$$

When $\delta \rightarrow 0$, the set $\Lambda_{\underline{\delta}}\left(\Gamma_{\mathbf{e}}\right)$ tends to $\Lambda_{\underline{0}}\left(\Gamma_{\mathbf{e}}\right)$.

The exponents and singular spaces associated with $\Delta_{\mu}$ are denoted by $\Lambda_{\mu}\left(\Gamma_{\mathbf{e}}\right)$ and $Z^{\lambda}\left(\Gamma_{\mathbf{e}}, \mu\right)$, according to Section 4.2 (iii).

\section{CORNER AND EDGE SINGULARITIES OF THE EDDY CURRENT PROBLEM}

The singularities of the solution $\mathbf{E}^{\delta}$ of problem (16) for $\delta>0$ are those of a Maxwell transmission problem, very similar to that investigated in [17]. Here, we concentrate on the solution of the eddy current problem $\mathbf{E}=\mathbf{E}^{0}$, which is also the solution of the regularized variational formulation $(21)$ for $\delta=0$, i.e.

$$
\text { Find } \mathbf{E} \in \mathbf{Y}(\Omega) \quad \text { s. t. } \quad a_{R}^{0}(\mathbf{E}, \mathbf{v})=-i \omega\left(\mathbf{j}_{0}, \mathbf{v}\right)_{\Omega_{C}}, \quad \forall \mathbf{v} \in \mathbf{Y}(\Omega)
$$

In this section we describe the corner and edge singularities of problem (51). These singularities are obtained as in $[15,17]$ with the necessary adaptations.

\subsection{Corner singularities}

Fix a corner $\mathbf{a}$ of $\Omega_{C}$ and denote by $\left(\rho_{\mathbf{a}}, \vartheta_{\mathbf{a}}\right)$ the spherical coordinates centered at $\mathbf{a}$. Denote furthermore by $\Gamma_{C, \mathbf{a}}\left(\right.$ resp. $\left.\Gamma_{E, \mathbf{a}}\right)$ the infinite polyhedral cone which coincides with $\Omega_{C}\left(\right.$ resp. $\left.\Omega_{E}\right)$ near a. Like in Section 4.3 , 
we denote by $\Gamma_{\mathbf{a}}$ the space $\mathbb{R}^{3}$ when we refer to its partition into $\Gamma_{C, \mathbf{a}}$ and $\Gamma_{E, \mathbf{a}}$. For shortness we now drop the index a. As usual we are looking for solutions of the homogeneous eddy current problem in the space

$$
\begin{gathered}
\mathbf{S}^{\lambda}(\Gamma)=\left\{\mathbf{u} \in \mathbf{X}_{l o c}(\Gamma): \operatorname{div} \mathbf{u}_{C} \in H_{l o c}^{1}\left(\Gamma_{C}\right), \operatorname{div} \mathbf{u}_{E} \in H_{l o c}^{1}\left(\Gamma_{E}\right),\right. \\
\left.\mathbf{u}(x)=\rho^{\lambda} \sum_{q=0}^{Q}(\log \rho)^{q} \mathbf{U}_{q}(\vartheta)\right\},
\end{gathered}
$$

the index loc meaning that the properties hold in all bounded domains far from $\mathbf{a}$. This means that we look for a non-polynomial solution $\mathbf{u} \in \mathbf{S}^{\lambda}(\Gamma)$ of (the last two boundary conditions may be justified by taking arbitrary right-hand sides $f \in L^{2}(\Omega)$ in (51), using arguments as in Th. 1.1 of [17])

$$
\begin{cases}\operatorname{curl}\left(\mu_{C}^{-1} \operatorname{curl} \mathbf{u}\right)-\nabla \operatorname{div} \mathbf{u}=0 & \text { in } \Gamma_{C}, \\ \operatorname{curl}\left(\mu_{E}^{-1} \operatorname{curl} \mathbf{u}\right)-\nabla \operatorname{div} \mathbf{u}=0 & \text { in } \Gamma_{E}, \\ \mathbf{u}_{C} \cdot \mathbf{n}=0 & \text { on } I:=\partial \Gamma_{C}=\partial \Gamma_{E}, \\ {[\mathbf{u} \times \mathbf{n}]=0} & \text { on } I, \\ {\left[\mu^{-1} \operatorname{curl} \mathbf{u} \times \mathbf{n}\right]=0, \quad[\operatorname{curl} \mathbf{u} \cdot \mathbf{n}]=0} & \text { on } I, \\ \partial_{n}\left(\operatorname{div} \mathbf{u}_{C}\right)=0 & \text { on } I, \\ \operatorname{div} \mathbf{u}_{E}=0 & \text { on } I .\end{cases}
$$

If a nontrivial solution exists then we say that $\lambda$ is an eddy current corner exponent.

For the sake of simplicity we assume that $\Gamma_{C}$ and $\Gamma_{E}$ are simply connected, the general case can be treated as in [15] and simply yields additional "topological" singular exponents.

As in $[15,17]$, this problem is split up into three subproblems by introducing the auxiliary unknowns

$$
\psi=\mu^{-1} \operatorname{curl} \mathbf{u}
$$

and

$$
q= \begin{cases}\operatorname{div} \mathbf{v}_{C} & \text { in } \Gamma_{C}, \\ \operatorname{div} \mathbf{v}_{E} & \text { in } \Gamma_{E} .\end{cases}
$$

With these notations, problem (52) is equivalent to looking for $q, \boldsymbol{\psi}, \mathbf{u}$, successive solutions of

$$
\begin{gathered}
\begin{cases}\Delta q=0 & \text { in } \Gamma_{C}, \\
\Delta q=0 & \text { in } \Gamma_{E}, \\
\partial_{n} q_{C}=0 & \text { on } I, \\
q_{E}=0 & \text { on } I,\end{cases} \\
\begin{cases}\operatorname{curl} \boldsymbol{\psi}=\nabla q, \operatorname{div}\left(\mu_{C} \boldsymbol{\psi}\right)=0 & \text { in } \Gamma_{C}, \\
\operatorname{curl} \boldsymbol{\psi}=\nabla q, \operatorname{div}\left(\mu_{E} \boldsymbol{\psi}\right)=0 & \text { in } \Gamma_{E}, \\
{[\boldsymbol{\psi} \times \mathbf{n}]=0, \quad[\mu \boldsymbol{\psi} \cdot \mathbf{n}]=0} & \text { on } I,\end{cases} \\
\begin{cases}\mathbf{c u r l} \mathbf{u}=\mu_{C} \boldsymbol{\psi}, \operatorname{div} \mathbf{u}=q & \text { in } \Gamma_{C}, \\
\operatorname{curl} \mathbf{u}=\mu_{E} \boldsymbol{\psi}, \operatorname{div} \mathbf{u}=q & \text { in } \Gamma_{E}, \\
\mathbf{u}_{C} \cdot \mathbf{n}=0 & \text { on } I, \\
{[\mathbf{u} \times \mathbf{n}]=0} & \text { on } I .\end{cases}
\end{gathered}
$$

This means that we have three types of singularities:

Type 1: $q=0, \boldsymbol{\psi}=0$ and $\mathbf{u}$ is a general solution of (55);

Type 2: $q=0, \boldsymbol{\psi}$ is a general solution of (54) and $\mathbf{u}$ a particular solution of (55);

Type 3: $q$ is a general solution of (53), $\boldsymbol{\psi}$ a particular solution of (54) and $\mathbf{u}$ a particular solution of (55). 
These three types of singularities may be described with the help of the corner singularities of the Neumann problem in $\Gamma_{C}$, of the Dirichlet problem in $\Gamma_{E}$ and of the transmission operator $\Delta_{\mu}$.

Since for our problem (51), $\operatorname{div} \mathbf{E}_{C}$ and $\operatorname{div} \mathbf{E}_{E}$ are regular, we do not describe the singularities of type 3 since they cannot occur for any solution of (51).

Let us start with the singularity of type 1 :

Lemma 5.1. Assume that $\lambda \neq-1$. Then $\mathbf{u} \in \mathbf{S}^{\lambda}(\Gamma)$ is a singularity of type 1 if and only if (i) or (ii) below holds.

(i) $\lambda+1$ belongs to $\Lambda^{\mathrm{Neu}}\left(\Gamma_{C}\right), \mathbf{u}=\nabla \Phi$, with $\Phi_{C} \in Z_{\mathrm{Neu}}^{\lambda+1}\left(\Gamma_{C}\right)$ and $\Phi_{E} \in S^{\lambda+1}\left(\Gamma_{E}\right)$ solution of

$$
\begin{cases}\Delta \Phi_{E}=0 & \text { in } \Gamma_{E} \\ \Phi_{E}=\Phi_{C} & \text { on } I\end{cases}
$$

(ii) $\lambda+1$ belongs to $\Lambda^{\operatorname{Dir}}\left(\Gamma_{E}\right), \mathbf{u}=\nabla \Phi$, with $\Phi_{C}=0$ and $\Phi_{E} \in Z_{\operatorname{Dir}}^{\lambda+1}\left(\Gamma_{E}\right)$.

Proof. As

$$
\begin{aligned}
& \operatorname{curl} \mathbf{u}_{C}=0 \text { in } \Gamma_{C}, \\
& \operatorname{curl} \mathbf{u}_{E}=0 \text { in } \Gamma_{E},
\end{aligned}
$$

there exists $\Phi_{C} \in S^{\lambda+1}\left(\Gamma_{C}\right)$ and $\Phi_{E} \in S^{\lambda+1}\left(\Gamma_{E}\right)$ such that

$$
\begin{array}{ll}
\mathbf{u}_{C}=\nabla \Phi_{C} & \text { in } \Gamma_{C} \\
\mathbf{u}_{E}=\nabla \Phi_{E} & \text { in } \Gamma_{E} .
\end{array}
$$

From (55) we deduce that

$$
\begin{cases}\Delta \Phi_{C}=0 & \text { in } \Gamma_{C} \\ \Delta \Phi_{E}=0 & \text { in } \Gamma_{E} \\ \partial_{n} \Phi_{C}=0 & \text { on } I \\ \Phi_{C}=\Phi_{E} & \text { on } I\end{cases}
$$

Then either $\Phi_{C}$ is not zero and we are in the case $(i)$ or $\Phi_{C}=0$ and we are in the case $(i i)$.

Lemma 5.2. Assume that $\lambda>0$. Then $\mathbf{u} \in \mathbf{S}^{\lambda}(\Gamma)$ is a singularity of type 2 if and only if $\lambda$ belongs to $\Lambda_{\mu}(\Gamma)$, $\boldsymbol{\psi}=\nabla \Psi$, with $\Psi \in Z^{\lambda}(\Gamma, \mu)$ and $\mathbf{u}$ given by

$$
\mathbf{u}=\frac{1}{\lambda+1}(\mu(\nabla \Psi \times \mathbf{x})+\nabla r)
$$

where $r_{C} \in S^{\lambda+1}\left(\Gamma_{C}\right), r_{E} \in S^{\lambda+1}\left(\Gamma_{E}\right)$ are solutions of

$$
\begin{cases}\Delta r_{C}=0 & \text { in } \Gamma_{C} \\ \Delta r_{E}=0 & \text { in } \Gamma_{E} \\ \partial_{n} r_{C}=-\mu_{C}\left(\nabla \Psi_{C} \times \mathbf{x}\right) \cdot \mathbf{n} & \text { on } I, \\ r_{C}=r_{E} & \text { on } I .\end{cases}
$$

Proof. As

$$
\begin{array}{ll}
\operatorname{curl} \psi_{C}=0 & \text { in } \Gamma_{C}, \\
\operatorname{curl} \psi_{E}=0 & \text { in } \Gamma_{E},
\end{array}
$$


there exists $\Psi_{C} \in S^{\lambda}\left(\Gamma_{C}\right)$ and $\Psi_{E} \in S^{\lambda}\left(\Gamma_{E}\right)$ such that

$$
\begin{array}{ll}
\psi_{C}=\nabla \Psi_{C} & \text { in } \Gamma_{C} \\
\psi_{E}=\nabla \Psi_{E} & \text { in } \Gamma_{E}
\end{array}
$$

From (54) we deduce that

$$
\begin{cases}\operatorname{div}\left(\mu_{C} \nabla \Psi_{C}\right)=0 & \text { in } \Gamma_{C} \\ \operatorname{div}\left(\mu_{E} \nabla \Psi_{E}\right)=0 & \text { in } \Gamma_{E} \\ {[\Psi]=0, \quad\left[\mu \partial_{n} \Psi\right]=0} & \text { on } I\end{cases}
$$

This means that $\Psi \in Z^{\lambda}(\Gamma, \mu)$.

Now we readily check that $\mathbf{u}$ in the form (57) is solution of (55) if and only if $r$ is solution of (58), whose solution exists by Theorem 4.14 of [26].

Lemma 5.3. (i) $\lambda=-1$ is not a corner singularity of type 1 .

(ii) $\lambda=0$ is not a corner singularity of type 2 .

Proof.

(i) If $\mathbf{u}$ is a singularity of type 1 for $\lambda=-1$, then $\mathbf{u}_{C}$ is a singularity of type 1 for $\lambda=-1$ for the Maxwell system in $\Gamma_{C}$ with the boundary condition $\mathbf{u}_{C} \cdot \mathbf{n}=0$ on $I$. Therefore by Lemma 7.8 of [15] $\mathbf{u}_{C}=0$. With this information, $\mathbf{u}_{E}$ is now a singularity of type 1 for $\lambda=-1$ for the Maxwell system in $\Gamma_{E}$ with the boundary condition $\mathbf{u}_{E} \times \mathbf{n}=0$ on $I$. Again by Lemma 7.8 of [15] we get $\mathbf{u}_{E}=0$.

(ii) If $\mathbf{u}$ is a singularity of type 2 for $\lambda=0$, then $\boldsymbol{\psi}=\mu^{-1}$ curl $\mathbf{u}_{C}$ is a singularity of type 1 for $\lambda=-1$ for the Maxwell interface system in $\mathbb{R}^{3}$ with the parameter $\mu$. Therefore by Lemma 5.4 of [17] we get $\boldsymbol{\psi}=0$.

Since the singularities of our problem (51) have to be locally in $\mathbf{X}(\Gamma)$ with a piecewise smooth divergence, among the singular exponents described above, we select the subset $\Lambda_{\mathbf{a}}$ of $\lambda>-\frac{3}{2}$ such that there exists $\mathbf{u} \in \mathbf{S}^{\lambda}(\Gamma)$ solution of $(52)$ such that

$$
\chi \mathbf{u} \in \mathbf{X}(\Gamma),
$$

where $\chi$ is a cut-off function equal to 1 near a. This last condition implies the following constraints for our two types of singularities (see [17]):

Type 1: $\lambda+1 \in \Lambda^{\mathrm{Neu}}\left(\Gamma_{C}\right)$ or $\lambda+1 \in \Lambda^{\operatorname{Dir}}\left(\Gamma_{E}\right)$ and since $\Lambda^{\operatorname{Dir}}\left(\Gamma_{E}\right) \cap[-1,0]$ and $\Lambda^{\mathrm{Neu}}\left(\Gamma_{C}\right) \cap[-1,0]$ are empty, by Lemma 5.3 we get $\lambda>-1$;

Type 2: $\lambda \in \Lambda_{\mu}(\Gamma)$ and by the condition $\operatorname{curl}(\chi \mathbf{u}) \in\left[L^{2}\left(\mathbb{R}^{3}\right)\right]^{3}$, we get $\lambda>-\frac{1}{2}$. By Lemma 5.3 and the fact that $\Lambda_{\mu}(\Gamma) \cap[-1,0]$ is empty, we get $\lambda>0$.

In conclusion we have

$$
\begin{aligned}
\Lambda_{\mathbf{a}}=\left\{\lambda>-1: \lambda+1 \in \Lambda^{\mathrm{Neu}}\left(\Gamma_{C}\right)\right\} & \cup\left\{\lambda>-1: \lambda+1 \in \Lambda^{\operatorname{Dir}}\left(\Gamma_{E}\right)\right\} \\
& \cup\left\{\lambda>0: \lambda \in \Lambda_{\mu}(\Gamma)\right\} .
\end{aligned}
$$

\subsection{Edge singularities}

Fix an edge e of $\Omega_{C}$ and denote by $\Gamma_{C} \times \mathbb{R}\left(\right.$ resp. $\Gamma_{E} \times \mathbb{R}$ ) the infinite polyhedral cone which coincides with $\Omega_{C}$ (resp. $\Omega_{E}$ ) near e ( $\Gamma_{C}$ and $\Gamma_{E}$ are then two-dimensional sectors). Denote by $(r, \theta, z)$ the cylindrical coordinates along e. As before we are looking for solutions of the homogeneous eddy current problem (52) in $\Gamma_{C} \times \mathbb{R}$ and $\Gamma_{E} \times \mathbb{R}$. Now $\Gamma$ refers to $\mathbb{R}^{2}$ with its partition into the two sectors $\Gamma_{C}$ and $\Gamma_{E}$, cf Section 4.4. Writing $\mathbf{u}=(\mathbf{v}, w)$, where $\mathbf{v}$ are the first two components of $\mathbf{u}$ in the Cartesian coordinates $\left(x_{1}, x_{2}, x_{3}\right)$ (according to the above notation, the $x_{3}$-axis contains the edge $\mathbf{e}$ ), the system (52) is split up into the following two independent 
problems in $\mathbb{R}^{2}$ :

$$
\begin{aligned}
& \begin{cases}\operatorname{curl}\left(\mu_{C}^{-1} \operatorname{curl} \mathbf{v}\right)-\nabla \operatorname{div} \mathbf{v}=0 & \text { in } \Gamma_{C} \\
\mathbf{c u r l}\left(\mu_{E}^{-1} \operatorname{curl} \mathbf{v}\right)-\nabla \operatorname{div} \mathbf{v}=0 & \text { in } \Gamma_{E}, \\
\mathbf{v}_{C} \cdot \mathbf{n}=0 & \text { on } I:=\partial \Gamma_{C}=\partial \Gamma_{E}, \\
{[\mathbf{v} \times \mathbf{n}]=0} & \text { on } I \\
{\left[\mu^{-1} \operatorname{curl} \mathbf{v} \times \mathbf{n}\right]=0} & \text { on } I \\
\partial_{n}\left(\operatorname{div} \mathbf{v}_{C}\right)=0 & \text { on } I \\
\operatorname{div} \mathbf{v}_{E}=0 & \text { on } I .\end{cases} \\
& \begin{cases}\operatorname{div}\left(\mu_{C}^{-1} \nabla w_{C}\right)=0 & \text { in } \Gamma_{C} \\
\operatorname{div}\left(\mu_{E}^{-1} \nabla w_{E}\right)=0 & \text { in } \Gamma_{E} \\
{[w]=0,\left[\mu^{-1} \partial_{n} w\right]=0} & \text { on } I .\end{cases}
\end{aligned}
$$

Problem (60) is a standard transmission problem whose set of singularities $\Lambda_{\mu^{-1}}(\Gamma)=\Lambda_{\mu}(\Gamma)$ (see Lem. 6.2 of [17]) were described in Section 4. Problem (59) is a two-dimensional eddy current problem whose singularities may be described as in 3D, by introducing the auxiliary unknowns $\psi=\mu^{-1}$ curl $\mathbf{v}$ and

$$
q= \begin{cases}\operatorname{div} \mathbf{v} & \text { in } \Gamma_{C} \\ \operatorname{div} \mathbf{v} & \text { in } \Gamma_{E}\end{cases}
$$

As before, singularities of type 1, 2 and 3 then appear. We can show that singularities of type 2 do not exist (compare with $[15,17]$ ), singularities of type 3 are not studied for the same reason as before, while singularities of type 1 are analyzed exactly as in Lemma 5.1 .

In conclusion we can state the following result.

Lemma 5.4. The set $\Lambda_{\mathbf{e}}$ of edge exponents associated with $\mathbf{e}$ is given by

$$
\begin{aligned}
\Lambda_{\mathbf{e}}=\left\{\lambda>-1: \lambda+1 \in \Lambda^{\mathrm{Neu}}\left(\Gamma_{C}\right)\right\} & \cup\left\{\lambda>-1: \lambda+1 \in \Lambda^{\mathrm{Dir}}\left(\Gamma_{E}\right)\right\} \\
& \cup\left\{\lambda>0: \lambda \in \Lambda_{\mu}(\Gamma)\right\} .
\end{aligned}
$$

If $\lambda \notin \mathbb{N} \backslash\{0\}$, then the associated singular function $\mathbf{u}=(\mathbf{v}, w)$ is as follows:

- If $\lambda+1 \in \Lambda^{\mathrm{Neu}}\left(\Gamma_{C}\right)$, then $w=0$,

$$
\mathbf{v}_{C}=\nabla\left(r^{\lambda+1} \varphi_{C}\right)
$$

with $\varphi_{C}(\theta)=\cos ((\lambda+1) \theta)$ (the half-lines $\theta=0$ and $\theta=\omega_{C}$ are assumed to be the interfaces between $\Gamma_{C}$ and $\Gamma_{E}$, the interior opening of $\Gamma_{C}\left(\right.$ resp. $\left.\Gamma_{E}\right)$ is then $\omega_{C}\left(\right.$ resp. $\left.\omega_{E}=2 \pi-\omega_{C}\right)$ ), and if $\frac{\omega_{E}}{\omega_{C}}$ is not a rational number, then

$$
\mathbf{v}_{E}=\nabla\left(r^{\lambda+1} \varphi_{E}\right)
$$

with $\varphi_{E}(\theta)=c_{1} \cos ((\lambda+1) \theta)+c_{2} \sin ((\lambda+1) \theta)$, for some (explicit) constants $c_{1}$ and $c_{2}$. If $\frac{\omega_{E}}{\omega_{C}}$ is a rational number, then a logarithmic term possibly appears in the expression of $\mathbf{v}_{E}$;

- if $\lambda+1 \in \Lambda^{\operatorname{Dir}}\left(\Gamma_{E}\right)$, then $w=0, \mathbf{v}_{C}=0$ and

$$
\mathbf{v}_{E}=\nabla\left(r^{\lambda+1} \varphi_{E}\right)
$$

with $\varphi_{E}(\theta)=\sin \left((\lambda+1)\left(\theta-\omega_{C}\right)\right)$;

- if $\lambda \in \Lambda_{\mu}(\Gamma)$, then $\mathbf{v}=0$ and $w=r^{\lambda} \varphi$, with $\varphi$ an eigenvector of problem (42) for $\alpha=\mu$, associated with the eigenvalue $\nu=\lambda^{2}$. 


\section{Regularity AND SINGULARITy RESUlts FOR THE EDDY CURRENT PROBLEM}

We describe the regularity as well as singular decompositions of any solution $\mathbf{E}$ of the regularized problem (51) with a source current density $\mathbf{j}_{0}$ such that

$$
\operatorname{div} \mathbf{j}_{0}=0, \quad \operatorname{supp} \mathbf{j}_{0} \subset \Omega_{C}, \quad \mathbf{j}_{0} \in\left[H^{s-1}\left(\Omega_{C}\right)\right]^{3} \text { for } s \geq 1 .
$$

These results are based on the knowledge of corner and edge singularities described in the previous section and rely on the application of Mellin techniques as in [15,17].

\subsection{Regularity}

For any corner a in the interface $B$ introduce

$$
\begin{aligned}
& \lambda_{C, \mathbf{a}}^{\mathrm{Neu}}=\min \left\{\lambda>0: \lambda \in \Lambda^{\mathrm{Neu}}\left(\Gamma_{C, \mathbf{a}}\right)\right\}, \\
& \lambda_{E, \mathbf{a}}^{\mathrm{Dir}}=\min \left\{\lambda>0: \lambda \in \Lambda^{\operatorname{Dir}}\left(\Gamma_{E, \mathbf{a}}\right)\right\}, \\
& \lambda_{\mu, \mathbf{a}}=\min \left\{\lambda>0: \lambda \in \Lambda_{\mu}\left(\Gamma_{\mathbf{a}}\right)\right\}
\end{aligned}
$$

where $\Lambda_{\mu}\left(\Gamma_{\mathbf{a}}\right)$ is defined in Section 4.3 for the subdivision of $\mathbb{R}^{3}$ into $\Gamma_{C, \mathbf{a}}$ and $\Gamma_{E, \mathbf{a}}$. Similarly for any edge e $\subset B$ define

$$
\lambda_{\mu, \mathbf{e}}=\min \left\{\lambda>0: \lambda \in \Lambda_{\mu}\left(\Gamma_{\mathbf{e}}\right)\right\}
$$

Now we can set

$$
\begin{aligned}
\tau_{\mathbf{e}}^{(1)} & :=\min \left(\frac{\pi}{\omega_{C, \mathbf{e}}}, \frac{\pi}{\omega_{E, \mathbf{e}}}\right), \\
\tau_{\mathbf{a}}^{(1)} & :=\min \left(\lambda_{C, \mathbf{a}}^{\text {Neu }}, \lambda_{E, \mathbf{a}}^{\text {Dir }}\right), \\
\tau^{(1)} & :=\min \left(\min _{\mathbf{e}} \tau_{\mathbf{e}}^{(1)}, \frac{1}{2}+\min _{\mathbf{a}} \tau_{\mathbf{a}}^{(1)}\right), \\
\tau_{C}^{(1)} & :=\min \left(\min _{\mathbf{e}} \frac{\pi}{\omega_{C, \mathbf{e}}}, \frac{1}{2}+\min _{\mathbf{a}} \lambda_{C, \mathbf{a}}^{\text {Neu }}\right), \\
\tau^{(2)} & :=\min \left(\min _{\mathbf{e}} \lambda_{\mu, \mathbf{e}}, \frac{1}{2}+\min _{\mathbf{a}} \lambda_{\mu, \mathbf{a}}\right),
\end{aligned}
$$

when $\omega_{C, \mathbf{e}}$ is the opening of $\Omega_{C}$ along $\mathbf{e}$ and $\omega_{E, \mathbf{e}}=2 \pi-\omega_{C, \mathbf{e}}$ is the opening of $\Omega_{E}$ along $\mathbf{e}$

Then we have

Theorem 6.1. Let $\mathbf{E} \in \mathbf{Y}(\Omega)$ be a solution of problem (51) with $\mathbf{j}_{0}$ satisfying (61) for $s \geq 1$. Then we have

$$
\begin{aligned}
& \mathbf{E}_{C} \in H^{\tau_{C}}\left(\Omega_{C}\right), \quad \forall \tau_{C}<\min \left(\tau_{C}^{(1)}, \tau^{(2)}+1, s+1\right), \\
& \mathbf{E}_{E} \in H^{\tau_{E}}\left(\Omega_{E}\right), \quad \forall \tau_{E}<\min \left(\tau^{(1)}, \tau^{(2)}+1, s+1\right) .
\end{aligned}
$$

\subsection{Singularities}

We start with a general result and then restrict ourselves to a particular case where there remain only singularities of type 1 .

The general result is proved exactly as in $[15,17]$ and can be stated as follows:

Theorem 6.2. Assume that $s \geq 1$ such that for all corners $\mathbf{a}, s-\frac{1}{2}$ does not belong to $\Lambda_{\mathbf{a}}$ and for all edges $\mathbf{e}$, $s$ does not belong to $\Lambda_{\mathbf{e}}$. Assume furthermore that the edge exponents in $[-1, s]$ are contained in an interval of 
length $<1$. Let $\mathbf{E} \in \mathbf{Y}(\Omega)$ be a solution of problem (51) with $\mathbf{j}_{0}$ satisfying (61) for this regularity exponent $s$. Then $\mathbf{E}$ admits the decomposition

$$
\mathbf{E}=\mathbf{E}^{(\mathrm{R})}+\mathbf{E}^{(\mathrm{S})}
$$

where the regular part satisfies

$$
\mathbf{E}_{C}^{(\mathrm{R})} \in H^{s+1}\left(\Omega_{C}\right) \quad \text { and } \quad \mathbf{E}_{E}^{(\mathrm{R})} \in H^{s+1}\left(\Omega_{E}\right) .
$$

On the other hand the singular part $\mathbf{E}^{(S)}$ has the standard structure

$$
\begin{aligned}
\mathbf{E}^{(\mathrm{S})}= & \sum_{\mathbf{a}} \sum_{\lambda \in \Lambda_{\mathbf{a}} \cap\left[-\frac{3}{2}, s-\frac{1}{2}\right]} \sum_{p} \gamma_{\mathbf{a}}^{\lambda, p} \chi_{\mathbf{a}}\left(\rho_{\mathbf{a}}\right) \mathbf{u}_{\mathbf{a}}^{\lambda, p}\left(\rho_{\mathbf{a}}, \vartheta_{\mathbf{a}}\right) \\
& +\sum_{\mathbf{e}} \sum_{\lambda \in \Lambda_{\mathbf{e}} \cap[-1, s]} \sum_{p} \mathcal{K}\left[\gamma_{\mathbf{e}}^{\lambda, p}\right] \chi_{\mathbf{e}}\left(\rho_{\mathbf{e}}\right) \mathbf{u}_{\mathbf{e}}^{\lambda, p}\left(\rho_{\mathbf{e}}, \theta_{\mathbf{e}}\right),
\end{aligned}
$$

where $\mathbf{u}_{\mathbf{a}}^{\lambda, p}$ (resp. $\mathbf{u}_{\mathbf{e}}^{\lambda, p}$ ) are the corner (resp. edge) singularities of type 1 or 2 described in the previous section, $\chi_{\mathbf{a}}\left(\right.$ resp. $\left.\chi_{\mathbf{e}}\right)$ is a smooth cut-off function equal to 1 near $\rho=0, \rho_{\mathbf{e}}=r_{\mathbf{e}} d_{\mathbf{e}}^{-1}$ when $d_{\mathbf{e}}$ is a smooth function which is equivalent to the distance of the endpoints of $\mathbf{e}, \mathcal{K}$ is a convolution operator (cf. $[15,17,18])$ and $\gamma_{\mathbf{a}}^{\lambda, p}$ $\left(\right.$ resp. $\gamma_{\mathbf{e}}^{\lambda, p}$ ) are real constant (resp. functions defined in the edge $\mathbf{e}$ and belonging to some weighted Sobolev spaces).

Exactly as in $[15,17]$, if one wants to eliminate the singularities of type 2 , we introduce a parameter $\tau \leq s$ satisfying

$$
\tau<\min \left\{\tau^{(1)}, \tau^{(2)}\right\}
$$

Using Lemmas 4.11 and 4.13 of [15], we obtain

Theorem 6.3. Let $\mathbf{E} \in \mathbf{Y}(\Omega)$ be a solution of problem (51) with $\mathbf{j}_{0}$ as in the introduction and the regularity $\mathbf{j}_{0} \in\left[H^{s-1}\left(\Omega_{C}\right)\right]^{3}$ with $s \geq 1$. Let $\tau \leq s$ satisfy $(65)$ and such that the edge exponents in $[-1, \tau]$ are contained in an interval of length $<1$. Then $\mathbf{E}$ admits the decomposition

$$
\mathbf{E}=\mathbf{E}^{(\mathrm{R})}+\nabla \Phi
$$

where the regular part satisfies

$$
\mathbf{E}_{C}^{(\mathrm{R})} \in H^{\tau+1}\left(\Omega_{C}\right) \quad \text { and } \quad \mathbf{E}_{E}^{(\mathrm{R})} \in H^{\tau+1}\left(\Omega_{E}\right),
$$

while $\Phi \in H^{1}(\Omega)$ satisfies

$$
\begin{aligned}
& \Delta \Phi_{C} \in H^{\tau}\left(\Omega_{C}\right) \\
& \partial_{n} \Phi_{C}=0 \text { on } B
\end{aligned}
$$

and

$$
\begin{aligned}
& \Delta \Phi_{E} \in H^{\tau}\left(\Omega_{E}\right), \\
& \Phi_{E}=\Phi_{C} \text { on } B .
\end{aligned}
$$

If $\tau=0$ the above theorem reduces to Theorem 3.1. For $\tau$ not necessarily equal to zero, as in that theorem, $\Phi_{C}$ has the singularities of the interior Neumann problem, while $\Phi_{E}$ has induced exterior singularities as well as exterior Dirichlet ones. 


\section{Continuity of the Singular functions in the EDdy CURREnt Limit}

If we put together:

(1) the result of Section 4.2 which yields the continuity of the singular exponents with respect to $\delta$ for the associated scalar problem;

(2) the common structure of Maxwell and eddy current singularities of type 1 (as gradients of scalar singularities);

(3) the similar structure of Maxwell and eddy current singularities of type 2 (compare our Lem. 5.2 with [17, Lem. 5.2]);

we may wonder whether it is possible to define a basis of singular fields $\mathbf{u}_{\mathbf{a}}^{\lambda, p}[\delta]$ and $\mathbf{u}_{\mathbf{e}}^{\lambda, p}[\delta]$ for the eddy current problem (51), $\delta=0$, and the Maxwell problem (21), $\delta>0$, which should be regular with respect to $\delta \in\left[0, \delta_{0}\right]$ and so that a decomposition like that of Theorem 6.2 holds with coefficients depending smoothly on $\delta$.

In this paper, we will not address this question in its full complexity, but show that it is possible to choose bases of singular functions in a regular way with respect to $\delta$, up to the limit $\delta=0$. This means that we have mainly to investigate the behavior of all singularities (i.e. in $S^{\lambda}(\Gamma)$ ) of the scalar problems $(40)$ when $\alpha=\alpha^{\delta}$ given by (43), as $\delta \rightarrow 0$. Similar questions are addressed in $[13,14,27]$. Since the direct application of these references is not straightforward, we are going to state the main steps of a relevant construction.

In the general case, we cannot exclude any of the phenomena such as "crossing" and "branching" that appear for singularity problems depending on a parameter. Since, in our situation, the coefficients are non-real, we may expect singularity exponents that have algebraic branch points for certain values of $\delta$, even for $\delta=0$, i.e. in the eddy current limit. We also can have changes of multiplicities, even for $\delta=0$, for example in the case where a singular exponent for the Neumann problem in $\Gamma_{C}$ coincides with a singular exponent for the Dirichlet problem in $\Gamma_{E}$.

In both these situations, any individual singular function of the transmission problem $\Delta_{\underline{\delta}}$ of the form $\rho^{\lambda_{\delta}} \psi_{\delta}(\vartheta)$ will converge to a singular function $\rho^{\lambda_{0}} \psi_{0}(\vartheta)$ of the limit problem, but the coefficients of a such singular function may be non-regular with respect to $\delta$ or even blow up for $\delta \rightarrow 0$. Clustering several singular functions together and choosing a basis depending analytically on $\delta$ as explained below in Section 7.2 will avoid such pathologies.

\subsection{Mellin symbols}

It is known from [23] that the corner singularities solution of (41) are produced by the poles of the associated Mellin symbol: Let us recall that the Mellin symbol of an operator $A$ homogeneous of degree $m$ with constant coefficients is $\lambda \mapsto \mathfrak{A}(\lambda)$ where

$$
A\left(\partial_{x}\right)=\rho^{-m} \mathfrak{A}\left(\vartheta ; \rho \partial_{\rho}, \partial_{\vartheta}\right) \text { and } \quad \mathfrak{A}(\lambda):=\mathfrak{A}\left(\vartheta ; \lambda, \partial_{\vartheta}\right) .
$$

Let us consider the situation of threedimensional cones $(d=3)$. The symbol associated with the operator (40) - see also (41), is

$$
\psi \longmapsto\left\{\begin{aligned}
L \psi_{C}-\lambda(\lambda+1) \psi_{C} & \text { in } \quad G_{C}, \\
L \psi_{E}-\lambda(\lambda+1) \psi_{E} & \text { in } \quad G_{E}, \\
\alpha_{C} \partial_{n_{C}} \psi_{C}+\alpha_{E} \partial_{n_{E}} \psi_{E} & \text { on } \quad J:=\partial G_{C}=\partial G_{E} .
\end{aligned}\right.
$$

Let us denote by $\mathfrak{M}_{\alpha}(\lambda)$ the operator (66) acting between function spaces:

$$
\mathfrak{M}_{\alpha}(\lambda): \mathbf{Z}\left(G_{C}, G_{E}\right) \longrightarrow L^{2}\left(G_{C}\right) \times L^{2}\left(G_{E}\right) \times H^{-1 / 2}(J),
$$

where the source space $\mathbf{Z}\left(G_{C}, G_{E}\right)$ is defined as

$$
\mathbf{Z}\left(G_{C}, G_{E}\right)=\left\{\varphi \in H^{1}\left(\mathbb{S}^{2}\right): \Delta \varphi_{C} \in L^{2}\left(G_{C}\right), \Delta \varphi_{E} \in L^{2}\left(G_{E}\right)\right\} .
$$

When $\alpha=\alpha^{\delta}$ is given by (43), we denote $\mathfrak{M}_{\alpha}(\lambda)$ by $\mathfrak{M}_{\underline{\delta}}(\lambda)$. 
We are going to prove that for all $\delta \in\left[0, \delta_{0}\right]$ there exists $\lambda$ such that $\mathfrak{M}_{\underline{\delta}}(\lambda)$ is invertible. Let $\left(f_{C}, f_{E}, g\right)$ belong to $L^{2}\left(G_{C}\right) \times L^{2}\left(G_{E}\right) \times H^{-1 / 2}(J)$ and let us fix $\lambda$ such that $-\lambda(\lambda+1)>0$.

- If $\delta=0$, we first solve the Neumann problem

$$
\begin{aligned}
& (L-\lambda(\lambda+1)) \psi_{C}=f_{C} \quad \text { in } \quad G_{C}, \\
& \alpha_{C} \partial_{n_{C}} \psi_{C}=g \quad \text { on } \quad J
\end{aligned}
$$

and then the Dirichlet problem

$$
\begin{aligned}
& (L-\lambda(\lambda+1)) \psi_{E}=f_{E} \quad \text { in } G_{E}, \\
& \psi_{E}=\left.\psi_{C}\right|_{J} \quad \text { on } \quad J .
\end{aligned}
$$

- If $\delta>0$, we use a variational formulation as in $(42): \forall \varphi \in H^{1}\left(\mathbb{S}^{d-1}\right)$,

$$
\int_{\mathbb{S}^{d-1}} \alpha \nabla \psi \cdot \nabla \varphi-\lambda(\lambda+1) \int_{\mathbb{S}^{d-1}} \alpha \psi \varphi=\int_{G_{C}} f_{C} \varphi_{C}+\int_{G_{E}} f_{E} \varphi_{E}+\int_{J} g \psi .
$$

Since the right hand side is clearly in $H^{-1}\left(\mathbb{S}^{d-1}\right)$, the coerciveness yields a unique solution.

The analytic Fredholm theorem yields that for all $\delta \in\left[0, \delta_{0}\right], \lambda \mapsto \mathfrak{M}_{\underline{\delta}}(\lambda)^{-1}$ is meromorphic. As the dependency of the symbol on $\delta$ is analytic, such is also the case for its inverse.

\subsection{Stable bases for singularities}

Let $\delta \in\left[0, \delta_{0}\right]$ be fixed. We recall that we have denoted by $\Lambda_{\underline{\delta}}(\Gamma)$ the set of the singular exponents of the operator $\Delta_{\underline{\delta}}$ (transmission or coupling).

The singular exponents in $\Lambda_{\underline{\delta}}(\Gamma)$ coincide with the poles $\lambda_{0}$ of $\mathfrak{M}_{\underline{\delta}}(\lambda)^{-1}$. Moreover the corresponding space of singularities $Z^{\lambda_{0}}(\Gamma ; \underline{\delta})$ (the space of solutions in $S^{\lambda_{0}}(\Gamma)$ of $(41)$ for $\alpha=\alpha^{\delta}$ ) is also given by a Cauchy residue formula:

$$
Z^{\lambda_{0}}(\Gamma ; \underline{\delta})=\left\{\Psi: \exists F \in \mathcal{O}\left(D\left(\lambda_{0}\right)\right), \quad \Psi=\frac{1}{2 i \pi} \int_{\partial D\left(\lambda_{0}\right)} \rho^{\lambda} \mathfrak{M}_{\underline{\delta}}(\lambda)^{-1} F(\lambda) \mathrm{d} \lambda\right\}
$$

where

- $D\left(\lambda_{0}\right)$ is a disc in the complex plane centered in $\lambda_{0}$ and not containing any other pole of $\mathfrak{M}_{\underline{\delta}}(\lambda)^{-1}$;

- The notation $F \in \mathcal{O}\left(D\left(\lambda_{0}\right)\right)$ means that $\lambda \mapsto F(\lambda)$ is holomorphic in a neighborhood of $\overline{D\left(\lambda_{0}\right)}$ with values in the target space $L^{2}\left(G_{C}\right) \times L^{2}\left(G_{E}\right) \times H^{-1 / 2}(J)$.

Note that if $D$ contains several elements of $\Lambda_{\underline{\delta}}(\Gamma)$, but $\partial D \cap \Lambda_{\underline{\delta}}(\Gamma)$ is empty, then

$$
\left\{\Psi: \exists F \in \mathcal{O}(D), \quad \Psi=\frac{1}{2 i \pi} \int_{\partial D} \rho^{\lambda} \mathfrak{M}_{\underline{\delta}}(\lambda)^{-1} F(\lambda) \mathrm{d} \lambda\right\}=\bigoplus_{\lambda \in \Lambda_{\underline{\underline{\varepsilon}}}(\Gamma) \cap D} Z^{\lambda}(\Gamma ; \underline{\delta}) .
$$

The smooth dependency on $\delta$ of $\mathfrak{M}_{\underline{\delta}}(\lambda)^{-1}$ implies the principle of smooth dependency of the singular spaces $Z^{\lambda}(\Gamma ; \underline{\delta})$ on $\delta$ in the following sense: Since we are interested in the eddy current limit let us consider a pole $\lambda_{0}$ of $\mathfrak{M}_{\underline{0}}(\lambda)^{-1}$ and a disc $D=D\left(\lambda_{0}\right)$ such that $\lambda_{0}$ is the only pole of $\mathfrak{M}_{0}(\lambda)^{-1}$ in $\bar{D}$. There exists $\delta\left(\lambda_{0}\right)$ such that for all $\delta \in\left[0, \delta\left(\lambda_{0}\right)\right]$ the symbols $\mathfrak{M}_{\underline{\delta}}(\lambda)^{-1}$ are invertible on $\partial D$. Then the spaces

$$
\left\{\Psi: \exists F \in \mathcal{O}(D), \quad \Psi=\frac{1}{2 i \pi} \int_{\partial D} \rho^{\lambda} \mathfrak{M}_{\underline{\delta}}(\lambda)^{-1} F(\lambda) \mathrm{d} \lambda\right\}
$$


depend smoothly on $\delta$ up to the limit $\delta=0$. In other words

$$
\bigoplus_{\lambda \in \Lambda_{\underline{\underline{\varepsilon}}}(\Gamma) \cap D} Z^{\lambda}(\Gamma ; \underline{\delta}) \quad \underset{\delta \rightarrow 0}{\longrightarrow} Z^{\lambda_{0}}(\Gamma ; \underline{0}) .
$$

Indeed, this statement shows the necessity of keeping together some clusters of poles.

We obtain easily a basis depending smoothly on $\delta$ : Choose $F^{1}, \ldots, F^{m}$ in $\mathcal{O}(D)$ such that

$$
\Psi_{0}^{n}(\rho, \vartheta):=\frac{1}{2 i \pi} \int_{\partial D} \rho^{\lambda} \mathfrak{M}_{\underline{0}}(\lambda)^{-1} F^{n}(\lambda) \mathrm{d} \lambda, \quad n=1, \ldots, m
$$

is a basis of $Z^{\lambda_{0}}(\Gamma ; \underline{0})$. Then, for $\delta$ small enough the functions

$$
\Psi_{\delta}^{n}(\rho, \vartheta):=\frac{1}{2 i \pi} \int_{\partial D} \rho^{\lambda} \mathfrak{M}_{\underline{\delta}}(\lambda)^{-1} F^{n}(\lambda) \mathrm{d} \lambda, \quad n=1, \ldots, m
$$

are a basis of $\bigoplus_{\lambda \in \Lambda_{\underline{\delta}}(\Gamma) \cap D} Z^{\lambda}(\Gamma ; \underline{\delta})$. The mappings $\delta \mapsto \Psi_{\delta}^{n}$ are analytic with respect to $\delta \in\left[0, \delta_{0}\right]$.

\subsection{Simple singularities}

If the dimension of $Z^{\lambda_{0}}(\Gamma ; \underline{0})$ is 1 , or more generally if $\delta=0$ is not a point of crossing or branching for the singularities, then the behavior of individual singular functions is very simple indeed. Let us consider the two typical situations where this happens:

(1) if $\lambda_{0} \in \Lambda^{\operatorname{Dir}}\left(\Gamma_{E}\right)$ is a simple eigenvalue and such that $\lambda_{0} \notin \Lambda^{\mathrm{Neu}}\left(\Gamma_{C}\right)$, then we can find a unique $\lambda_{\delta} \in \Lambda_{\underline{\delta}}(\Gamma)$ such that $\delta \mapsto \lambda_{\delta}$ is analytic for $\delta$ in a neighborhood of 0 . If we fix a $\rho^{\lambda_{0}} \psi_{0}$ in the singular space $\bar{Z}_{\text {Dir }}^{\lambda_{0}}\left(\Gamma_{E}\right)$, then we find a generator $\rho^{\lambda_{\delta}} \psi_{\delta}$ of $Z^{\lambda}(\Gamma ; \underline{\delta})$ such that $\delta \mapsto \psi_{\delta}$ is analytic for $\delta$ small enough. Then $\psi_{\delta, C} \rightarrow 0$ on $G_{C}$ in $H^{1}\left(G_{C}\right)$ and $\psi_{\delta, E} \rightarrow \psi_{0, E}$ on $G_{E}$ in $H^{1}\left(G_{E}\right)$;

(2) if $\lambda_{0} \in \Lambda^{\mathrm{Neu}}\left(\Gamma_{C}\right) \backslash \Lambda^{\operatorname{Dir}}\left(\Gamma_{E}\right)$, we have a singular function $\rho^{\lambda_{\delta}} \psi_{\delta}$ with $\delta \mapsto \lambda_{\delta}$ and $\delta \mapsto \psi_{\delta}$ analytic. Here $\rho^{\lambda_{0}} \psi_{0, C}$ is a singular function of the Neumann problem in $\Gamma_{C}$ and $\psi_{0, E}$ is the harmonic extension of $\psi_{0, C}$ to $\mathbb{S}^{2}$.

Situation 1, resp. 2, occurs for the first corner singularity of the eddy current problem where $\Omega_{C}$ has a corner like a cube, resp. like the exterior of a cube.

Situation 1 or 2 always occurs for the first edge singularity where the exponent is

$$
\lambda=\min \left\{\frac{\pi}{\omega_{C}}, \frac{\pi}{\omega_{E}}\right\}-1
$$

because $\pi / \omega_{C}$ and $\pi / \omega_{E}=\pi /\left(2 \pi-\omega_{C}\right)$ never coincide. Although unpredictable in general, the simplicity of limiting exponents of singularity is generic.

Acknowledgements. This work was completed while the first two authors were visiting the Newton Institute for Mathematical Sciences, Cambridge, UK.

\section{REFERENCES}

[1] A. Alonso and A. Valli, A domain decomposition approach for heterogeneous time-harmonic Maxwell equations. Comput. Methods Appl. Mech. Engrg 143 (1997) 97-112.

[2] A. Alonso-Rodriguez, F. Fernandes and A. Valli, Weak and strong formulations for the time-harmonic eddy-current problem in general domains. Report UTM. Dipartimento di Matematica, Univ. di Trento, Italy 603 (2001).

[3] H. Ammari, A. Buffa and J.-C. Nédélec, A justification of eddy currents model for the Maxwell equations. SIAM J. Appl. Math. 60 (2000) 1805-1823. 
[4] C. Amrouche, C. Bernardi, M. Dauge and V. Girault, Vector potentials in three-dimensional nonsmooth domains. Math. Methods Appl. Sci. 21 (1998) 823-864.

[5] F. Assous, P. Ciarlet Jr. and E. Sonnendrucker, Resolution of the Maxwell equations in a domain with reentrant corners. RAIRO Modél. Math. Anal. Numér. 32 (1998) 359-389.

[6] R. Beck, R. Hiptmair, R.H.W. Hoppe and B. Wohlmuth, Residual based a posteriori error estimators for eddy current computation. ESAIM: M2AN 34 (2000) 159-182.

[7] M. Birman and M. Solomyak, $L^{2}$-theory of the Maxwell operator in arbitrary domains. Russian Math. Surveys 42 (1987) $75-96$.

[8] M. Birman and M. Solomyak, On the main singularities of the electric component of the electro-magnetic field in regions with screens. St. Petersburg. Math. J. 5 (1993) 125-139.

[9] A.-S. Bonnet-Ben Dhia, C. Hazard and S. Lohrengel, A singular field method for the solution of Maxwell's equations in polyhedral domains. SIAM J. Appl. Math. 59 (1999) 2028-2044.

[10] A. Bossavit, Two dual formulations of the 3D eddy-current problem. COMPEL 4 (1985) 103-116.

[11] A. Bossavit, Electromagnétisme en vue de la modélisation. Springer-Verlag (1993).

[12] D. Colton and R. Kress, Integral equation methods in scattering theory. John Wiley \& Sons, Inc., New York, Pure Appl. Math. (1983).

[13] M. Costabel and M. Dauge, Singularités d'arêtes pour les problèmes aux limites elliptiques, in Journées "Équations aux Dérivées Partielles" (Saint-Jean-de-Monts, 1992), Exp. No. IV, 12 p. École Polytech., Palaiseau (1992).

[14] M. Costabel and M. Dauge, Stable asymptotics for elliptic systems on plane domains with corners. Comm. Partial Differential Equations 9 \& 10 (1994) 1677-1726.

[15] M. Costabel and M. Dauge, Singularities of Maxwell's equations on polyhedral domains. Arch. Rational Mech. Anal. 151 (2000) 221-276.

[16] M. Costabel and M. Dauge, Weighted regularization of Maxwell equations in polyhedral domains. A rehabilitation of nodal finite elements. Numer. Math. 93 (2002) 239-277.

[17] M. Costabel, M. Dauge and S. Nicaise, Singularities of Maxwell interface problems. ESAIM: M2AN 33 (1999) 627-649.

[18] M. Dauge, Elliptic boundary value problems on corner domains. Springer-Verlag, Berlin L.N. in Math. 1341 (1988).

[19] M. Dobrowolski, Numerical approximation of elliptic interface and corner problems. Habilitationsschrift, Bonn, Germany (1981).

[20] V. Girault and P.-A. Raviart, Finite element methods for Navier-Stokes equations. Springer-Verlag, Springer Ser. Comput. Math. 5 (1986).

[21] P. Grisvard, Elliptic problems in nonsmooth domains. Monographs and Studies in Mathematics. Pitman, Boston 24 (1985).

[22] R. Hiptmair, Symmetric coupling for eddy currents problems. SIAM J. Numer. Anal. 40 (2002) 41-65.

[23] V.A. Kondrat'ev, Boundary-value problems for elliptic equations in domains with conical or angular points. Trans. Moscow Math. Soc. 16 (1967) 227-313.

[24] D. Leguillon and E. Sanchez-Palencia, Computation of singular solutions in elliptic problems and elasticity. RMA 5. Masson, Paris (1991).

[25] D. Mercier, Minimal regularity of the solutions of some transmission problems. Math. Methods Appl. Sci. 26 (2003) 321-348.

[26] S. Nicaise, Polygonal interface problems. Peter Lang, Berlin (1993).

[27] S. Nicaise and A.-M. Sändig, General interface problems I,II. Math. Methods Appl. Sci. 17 (1994) 395-450.

[28] S. Nicaise and A.-M. Sändig, Transmission problems for the Laplace and elasticity operators: Regularity and boundary integral formulation. Math. Methods Appl. Sci. 9 (1999) 855-898.

[29] S. Nicaise, Edge elements on anisotropic meshes and approximation of the Maxwell equations. SIAM J. Numer. Anal. 39 (2001) 784-816.

[30] R. Picard, On the boundary value problems of electro- and magnetostatics. Proc. Roy. Soc. Edinburgh Sect. A 92 (1982) $165-174$. 